\title{
Bottom-quark forward-backward asymmetry, dark matter, and the LHC
}

\author{
Da Liu, ${ }^{1}$ Jia Liu, ${ }^{2, *}$ Carlos E. M. Wagner, ${ }^{1,2,3}$ and Xiao-Ping Wang ${ }^{1}$ \\ ${ }^{1}$ High Energy Physics Division, Argonne National Laboratory, Argonne, Illinois 60439, USA \\ ${ }^{2}$ Physics Department and Enrico Fermi Institute, University of Chicago, Chicago, Illinois 60637, USA \\ ${ }^{3}$ Kavli Institute for Cosmological Physics, University of Chicago, Chicago, Illinois 60637, USA
}

(Received 4 January 2018; published 15 March 2018)

\begin{abstract}
The LEP experiment at CERN provided accurate measurements of the $Z$ neutral gauge boson properties. Although all measurements agree well with the standard model (SM) predictions, the forward backward asymmetry of the bottom-quark remains almost $3 \sigma$ away from the SM value. We proposed that this anomaly may be explained by the existence of a new $U(1)_{D}$ gauge boson, which couples with opposite charges to the right-handed components of the bottom and charm quarks. Cancellation of gauge anomalies demands the presence of a vector-like singlet charged lepton as well as a neutral Dirac (or Majorana) particle that provides a dark matter candidate. Constraints from precision measurements imply that the mass of the new gauge boson should be around $115 \mathrm{GeV}$. We discuss the experimental constraints on this scenario, including the existence of a di-jet resonance excess at an invariant mass similar to the mass of this new gauge boson, observed in boosted topologies at the CMS experiment.
\end{abstract}

DOI: 10.1103/PhysRevD.97.055021

\section{INTRODUCTION}

The standard model (SM) provides an accurate description of all experimental observables. The discovery of a $125 \mathrm{GeV}$ resonance with properties consistent with a $125 \mathrm{GeV}$ Higgs boson [1,2] provides evidence of the realization of the Higgs mechanism as a source of gauge boson and fermion masses. However, the exact properties of the Higgs sector are still unknown. The minimal model postulates the existence of just one Higgs, transforming as a doublet under the gauge interactions. Precision measurements of the charged and neutral gauge boson properties [3] show the preference towards a doublet Higgs state. Similar properties would be obtained, however, if there were more than just one Higgs doublets. Finally, the presence of extra singlet scalar Higgs states is not constrained by these considerations.

Another outstanding question is the origin of the dark matter (DM) observed in astrophysical configurations. The standard model does not provide any DM candidate and its nature is unknown. Among the many DM candidates, weakly interactive massive particles (WIMPs) are particularly attractive since they can easily be incorporated in

\footnotetext{
*Corresponding author. liuj1@uchicago.edu

Published by the American Physical Society under the terms of the Creative Commons Attribution 4.0 International license. Further distribution of this work must maintain attribution to the author(s) and the published article's title, journal citation, and DOI. Funded by SCOAP .
}

beyond the SM scenarios. Moreover, it is well known that WIMPs with mass of the order of the weak scale and interactions of about the weak scale one provide a good candidate of thermal DM candidate [4].

Precision measurements of the gauge sector have shown agreement with expected SM properties at the per-mille level. Such a precision leads to sensitivity to radiative corrections which depend in a relevant way on the topquark and the Higgs mass. Among the many observables measured, the bottom forward-backward asymmetry measured at LEP presents a $3 \sigma$ deviation with respect to the values expected in the SM [3]. Although this deviation could be just due to statistical fluctuations, its nature is intriguing since it could be associated with a large correction to the right-handed bottom quark coupling to the $Z$ boson, which may only be explained by either mixing of the bottom-quark with additional (vectorlike) quarks, or by mixing of the $Z$ gauge boson with additional neutral gauge bosons. The first possibility led to the proposal of what are called beautiful-mirror scenarios [5], and their properties have been studied in detail [6-8]. The second possibility, namely the existence of additional gauge bosons contributing via mixing to a variation of the bottom quark coupling has also been explored, within the context of left-right models and warped extra dimensions $[9,10]$. In this article, we study the properties of a neutral gauge boson with preferential couplings to the bottom and charm quarks. We shall show that it leads naturally to the existence of a low energy spectrum that includes two Higgs doublets, a singlet, and a charged and a neutral vectorlike singlets, the latter being a good DM candidate. 
This article is organized as follows. In Sec. II, we describe the properties of the proposed SM gauge extension. We present the tree-level couplings of the new gauge boson to SM particles, as well as the necessary fermion content in order to cancel the gauge anomalies. The new Higgs bosons are introduced in order to induce the necessary mixing and provide masses to all chiral fermions in the theory. In Sec. III, we study the constraints on this model coming from precision electroweak measurements. In Sec. IV, we study the collider constraints on this model and in Sec. V we study the constraints coming from the requirement of obtaining the proper DM relic density without being in conflict with direct and indirect detection constraints. We reserve Sec. VI to our conclusions.

\section{A MODEL WITH TWO HIGGS DOUBLETS AND A SINGLET}

In this section, we shall describe the precise gauge extension of the SM we propose to explain the anomalous value of the bottom-quark forward-backward asymmetry. We consider a new gauge group $U(1)_{D}$ with gauge boson field $K_{\mu}$ [11], under which, the right-handed bottom and right-handed charm quark have opposite charge $\pm X$. This ensures the automatic cancellation of the $S U(3)_{c}^{2} \times$ $U(1)_{D}, U(1)_{D}^{3}$ gauge anomaly. In order to cancel the gauge anomalies involving the hyper-charge gauge field, we introduce two $S U(2)$ singlet SM-vector-like leptons $\chi_{1,2}$ with hyper charge -1 and 0 , where only the right-handed components are charged under $U(1)_{D}$, carrying charges $\pm X$, respectively. The neutral state $\chi_{2}$ will be naturally a dark matter candidate, provided we impose a $Z_{2}$ parity, under which $\chi_{2}$ transforms non-trivially while SM-particles are neutral under this symmetry transformations.

A modification of the forward-backward asymmetry, consistent with the one observed experimentally, may be obtained by a sizable variation of the coupling of the $Z$ to right-handed bottom quarks [5]. Such a variation of the $Z$ gauge boson couplings may be the result of mixing between the $Z$ and the $K$ gauge bosons. Such mixing may be induced by a new $S U(2)$ Higgs doublet $\Phi_{1}$ with hypercharge $Y=1 / 2$ and $U(1)_{D}$ charge equal to the $b_{R}$ one, which is needed to make sure that we obtain the enhanced $Z b_{R} \bar{b}_{R}$ coupling for $m_{K}>m_{Z}$.

The SM Higgs-like doublet which gives the other SM fermions and the gauge bosons masses will be denoted as $\Phi_{2}$. Another SM gauge singlet scalar $\Phi_{3}$ charged under $U(1)_{D}$ is needed to give mass to the $K$ gauge boson. It is clear that within this setup, we can not write down the normal Yukawa interaction for the bottom and charm quark directly. To solve the problem, we add two vector-like quarks $\psi_{b}, \psi_{c}$, which have the same SM charges as $b_{R}$ and $c_{R}$, but without $U(1)_{D}$ charge. The masses of the bottom and charm quarks are obtained by their mixing with the heavy vectorlike quarks, which is in the same spirit of partial compositeness [12]. The particle contents of our model and their gauge group charges are listed in Table I.
TABLE I. All particles with $S U(3)_{C} \times S U(2)_{L} \times U(1)_{Y} \times$ $U(1)_{D}$ charge specified. The anomaly-free condition is applied for this model. The $U(1)_{D}$ charge of $b_{R}, c_{R}, \chi_{1, R}$ and $\chi_{2, R}$ is determined by the anomaly-free condition. We choose $X=1$ for the model without loss of generality.

\begin{tabular}{lccrr}
\hline \hline Filed & $S U(3)_{C}$ & $S U(2)_{L}$ & $U(1)_{Y}$ & $U(1)_{D}$ \\
\hline$\Phi_{1}$ & 1 & 2 & $\frac{1}{2}$ & $\mathrm{X}$ \\
$\Phi_{2}$ & 1 & 2 & $\frac{1}{2}$ & 0 \\
$\Phi_{3}$ & 1 & 1 & 0 & $-\mathrm{X}$ \\
$b_{R}$ & 3 & 1 & $-\frac{1}{3}$ & $\mathrm{X}$ \\
$c_{R}$ & 3 & 1 & $\frac{2}{3}$ & $-\mathrm{X}$ \\
$\chi_{1, R}$ & 1 & 1 & -1 & $\mathrm{X}$ \\
$\chi_{2, R}$ & 1 & 1 & 0 & $-\mathrm{X}$ \\
$\chi_{1, L}$ & 1 & 1 & -1 & 0 \\
$\chi_{2, L}$ & 1 & 1 & 0 & 0 \\
$\psi_{b}$ & 3 & 1 & $-\frac{1}{3}$ & 0 \\
$\psi_{c}$ & 3 & 1 & $\frac{2}{3}$ & 0 \\
\hline \hline
\end{tabular}

The whole Lagrangian in our models can be written into three parts:

$$
\mathcal{L}=\mathcal{L}_{\Phi}+\mathcal{L}_{q}+\mathcal{L}_{\ell}
$$

where $\mathcal{L}_{\Phi, q, \ell}$ denotes that Lagrangian in the Higgs sector, the quark sector and the lepton sector respectively. For the Higgs part, the Lagrangian is simply as follows:

$\mathcal{L}_{\Phi}=\left|D_{\mu} \Phi_{1}\right|^{2}+\left|D_{\mu} \Phi_{2}\right|^{2}+\left|D_{\mu} \Phi_{3}\right|^{3}-V\left(\Phi_{i}, \Phi_{i}^{\dagger}\right)$,

where the covariant derivative is defined as:

$$
D_{\mu}=\partial_{\mu}-i g_{D} Y_{D} K_{\mu}-i g W_{\mu}^{a} \frac{\sigma^{a}}{2}-i g^{\prime} Y B_{\mu},
$$

where $K_{\mu}$ is the $U(1)_{X}$ gauge boson, $W_{\mu}^{a}$ are the SM $S U(2)_{L}$ gauge bosons and $B_{\mu}$ is the $U(1)_{Y}$ hypercharge gauge boson. The gauge bosons denoted without tildes are gauge eigenstates. After considering mixing effects, we shall later use tildes to denote mass eigenstates. For $W_{\mu}^{ \pm}$and the photon $A_{\mu}$, since they do not mix with $K_{\mu}$, the notation is the same as in the SM and there is no need to add tildes. The Higgs potential will be fully discussed in next subsection, and here we just assume that the fields associated with the three $C P$-even neutral Higgs bosons obtain vacuum expectation values (vev), i.e.

$$
\begin{aligned}
& \Phi_{1}=\left(\begin{array}{c}
h_{1}^{+} \\
\frac{1}{\sqrt{2}}\left(v_{1}+h_{1}^{0}+i a_{1}^{0}\right)
\end{array}\right), \\
& \Phi_{2}=\left(\begin{array}{c}
h_{2}^{+} \\
\frac{1}{\sqrt{2}}\left(v_{2}+h_{2}^{0}+i a_{2}^{0}\right)
\end{array}\right), \\
& \Phi_{3}=\frac{1}{\sqrt{2}}\left(v_{D}+h_{3}^{0}+i a_{3}^{0}\right) .
\end{aligned}
$$


The vev's do not break the electromagnetism symmetry, and $\Phi_{1}$ induce the mixing between the neutral massive gauge bosons $K_{\mu}$ and $Z_{\mu}$, which are proportional to $v_{1}^{2}$. Since the $W$ boson mass is not modified, the custodial symmetry is explicitly broken by the mixing and this will be reflected in $T$ parameter. The high-precision constraints on the $T$ parameter tell us that the mixing should be very small, which favors a small vev, $v_{1} \ll v_{2, D}$. For later convenience, it is useful to define the ratio angle $\beta$ :

$$
\tan \beta=\frac{v_{2}}{v_{1}},
$$

which controls the charged Higgs mixing by Goldstone equivalence theorem and has to be large. In this limit, the neutral $C P$-even Higgs $h_{2}^{0}$ will roughly be the SM-like $125 \mathrm{GeV}$ Higgs boson observed at the LHC $[1,13]$, and mixes with the $C P$-even Higgs boson $h_{1}^{0}$. The remaining physical charged Higgs and $C P$-odd Higgs bosons will be $\Phi_{1}$-like, while $h_{2}^{+}, a_{2}^{0}, a_{3}^{0}$ becomes the dominant longitudinal part of the massive $W, Z, K$ gauge bosons. $\Phi_{1}$-like physical Higgs will couple to SM gauge bosons and fermions suppressed by mixing angle to SM-like Higgs $\cot \beta$. The last $C P$-even Higgs boson will be $\Phi_{3}$-like and only couple largely to $U(1)_{D}$ charged particles and the $U(1)_{D}$ gauge boson $K_{\mu}$. As its vev $v_{D}$ is the source of bottom and charm masses, it couples with them proportional to their masses, i.e. $m_{b, c} / v_{D}$. In the absence of mixing with the other $C P$-even states it will be produced in bottom-fusion and gluon fusion processes and it will decay mostly to bottom quarks. Hence, provided the mixing with the SM-like Higgs boson is small, the LHC constraints on it are expected to be very weak.

The most general interactions in the quark sector are given by:

$$
\begin{aligned}
\mathcal{L}_{q}= & \sum_{q} i \bar{q} \not D q-m_{b, \psi} \bar{\psi}_{b} \psi_{b}-m_{c, \psi} \bar{\psi}_{c} \psi_{c} \\
& -\left(\bar{Q}_{L}^{i} y_{2 u}^{i j} \tilde{\Phi}_{2} u_{R}^{j}+\bar{Q}_{L}^{i} y_{2 d}^{i j} \Phi_{2} d_{R}^{j}+\text { H.c. }\right) \\
& -y_{2 b, \psi}^{i} \bar{Q}_{L}^{i} \Phi_{2} \psi_{b, R}-y_{2 c, \psi}^{i} \bar{Q}_{L}^{i} \tilde{\Phi}_{2} \psi_{c, R}-y_{3 b, \psi} \bar{\psi}_{b, L} \Phi_{3} b_{R} \\
& -y_{3 c, \psi} \bar{\psi}_{c, L} \Phi_{3}^{*} c_{R}+\text { H.c. },
\end{aligned}
$$

where $\tilde{\Phi}_{2}=i \sigma_{2} \Phi_{2}^{\dagger}, Q_{L}^{i}$ is the three family SM $S U(2)$ quark doublet and $u_{R}^{j}=\left(u_{R}, t_{R}\right), d_{R}^{j}=\left(d_{R}, s_{R}\right)$. The vev of $\Phi_{3}$ will induce the mixing between the right-handed bottom and charm quarks, $b_{R}, c_{R}$, and their corresponding vectorlike quark partner. As a result, the bottom and charm quarks obtain masses after electroweak spontaneous symmetry breaking (EWSB). In this sense, it is very similar to the partial compositeness scenario of the composite Higgs models except that our vectorlike quark partners can be fundamental. It is not difficult to embed our model to a composite Higgs model, where all the Higgs bosons are
Goldstone bosons associated with spontaneously broken global symmetry of a new strong sector.

As described above, the masses of the bottom and charm quark arise from the spontaneously broken $U(1)_{D}$ gauge symmetry and electroweak gauge symmetry, which can also been seen by integrating out the heavy vectorlike quark $\psi_{b, c}$ at the tree level using equation of motion:

$$
\begin{aligned}
\psi_{b, R}=-\frac{y_{3 b, \psi}}{m_{b, \psi}} \Phi_{3} b_{R}+\cdots, & \psi_{b, L}=-\frac{y_{2 b, \psi}^{i}}{m_{b, \psi}} \Phi_{2}^{\dagger} Q_{L}^{i}+\cdots, \\
\psi_{c, R}=-\frac{y_{3 c, \psi}}{m_{c, \psi}} \Phi_{3}^{*} b_{R}+\cdots, & \psi_{c, L}=-\frac{y_{2 c, \psi}^{i}}{m_{c, \psi}} \Phi_{2}^{\dagger} Q_{L}^{i}+\cdots,
\end{aligned}
$$

then we have the effective Yukawa interaction Lagrangian:

$$
\begin{aligned}
\mathcal{L}_{q}^{Y}= & -\left(\bar{Q}_{L}^{i} y_{2 u}^{i j} \tilde{\Phi}_{2} u_{R}^{j}+\bar{Q}_{L}^{i} y_{2 d}^{i j} \Phi_{2} d_{R}^{j}\right. \\
& +\frac{y_{2 b, \psi}^{i} y_{3 b, \psi}}{m_{b, \psi}} \bar{Q}_{L}^{i} \Phi_{2} \Phi_{3} b_{R} \\
& \left.+\frac{y_{2 c, \psi}^{i} y_{3 c, \psi}}{m_{c, \psi}} \bar{Q}_{L}^{i} \tilde{\Phi}_{2} \Phi_{3}^{*} c_{R}+\text { H.c. }\right),
\end{aligned}
$$

It is clear that the flavor interaction structure of $h_{2}^{0}$ is of SM-like and the effective Yukawa couplings may be diagonalized at the same time as the mass matrices. Although the last two terms in Eq. (8) can in principle induce flavor changing neutral current (FCNC) in the quark sector, it is very model dependent. In the following, we will assume the flavor-off-diagonal interactions are very small, which is equivalent to start with the Lagrangian with following parameters:

$$
y_{2 b, \psi}^{i}=\delta^{i 3} y_{2 b, \psi}, \quad y_{2 c, \psi}^{i}=\delta^{i 2} y_{2 c, \psi}, \quad y_{2 u}^{2 j}=0, \quad y_{2 d}^{3 j}=0 .
$$

For the leptons, we will focus on the third generation and similarly neglect off-diagonal terms between different generations. The Yukawa interaction Lagrangian reads:

$$
\begin{aligned}
\mathcal{L}_{\ell}^{Y}= & -y_{\tau} \bar{L}_{L, \tau} \Phi_{2} \tau_{R}-y_{\chi_{1}} \bar{\chi}_{1, L} \Phi_{3} \chi_{1, R}-y_{\chi_{2}} \bar{\chi}_{2, L} \Phi_{3}^{*} \chi_{2, R} \\
& -\frac{1}{2} M_{m} \bar{\chi}_{2, L} \chi_{2, L}^{c}-m_{\tau_{1}} \bar{\chi}_{1, L} \tau_{R}+\text { H.c. }
\end{aligned}
$$

where we have imposed the $Z_{2}$ parity for the neutral lepton $\chi_{2} \rightarrow-\chi_{2}$ and assumed that $\chi_{1}$ only mix with the third generation charged lepton $\tau_{R}$ by the direct Dirac mass $m_{\tau_{1}}$, which is the only source of $\chi_{1}$ decay. 


\section{A. The gauge sector}

In this subsection, we will discuss the mixing in the gauge sector and the couplings of the dark gauge boson. After the gauge symmetry breaking, the charged gauge boson sector is the same as SM with $v^{2}=v_{1}^{2}+v_{2}^{2}$ :

$$
m_{W}^{2}=\frac{1}{4} g^{2} v^{2}
$$

For the neutral sector, we first apply the rotation to transform $W_{3}, B$ gauge bosons into $Z, A$ gauge bosons as in the SM. The $\Phi_{1}$ is charged under both SM $S U(2)_{L} \times$ $U(1)_{Y}$ and $U(1)_{D}$, thus induces off-diagonal mass terms for $Z_{\mu}$ and $K_{\mu}$, but the photon state $A_{\mu}$ is not affected and stays massless, as it should be. Factoring out the photon state $A_{\mu}$, the $Z_{\mu}$ and $K_{\mu}$ will mix with each other and the mixing mass-square matrix is given by

$$
M_{V}^{2}=\left(\begin{array}{cc}
m_{Z}^{2} & -\frac{2 g_{D} c_{\beta}^{2}}{\sqrt{g^{2}+g^{2}}} m_{Z}^{2} \\
-\frac{2 g_{D} c_{\beta}^{2}}{\sqrt{g^{2}+g^{2}}} m_{Z}^{2} & m_{K}^{2}+\frac{4 g_{D}^{2} c_{\beta}^{2}}{g^{2}+g^{2}} m_{Z}^{2}
\end{array}\right),
$$

where we have defined:

$m_{Z}^{2}=\frac{\left(g^{2}+g^{\prime 2}\right) v^{2}}{4}, \quad c_{\beta}=\cos \beta=\frac{v_{1}}{v}, \quad m_{K}^{2}=g_{D}^{2} v_{D}^{2}$.

The matrix can be easily diagonalized by an $2 \times 2$ orthogonal matrix with mixing angle $\alpha$ :

$$
\left(\begin{array}{c}
Z_{\mu} \\
K_{\mu}
\end{array}\right)=\left(\begin{array}{cc}
\cos \alpha & \sin \alpha \\
-\sin \alpha & \cos \alpha
\end{array}\right)\left(\begin{array}{c}
\tilde{Z}_{\mu} \\
\tilde{K}_{\mu}
\end{array}\right),
$$

where $\tilde{Z}_{\mu}, \tilde{K}_{\mu}$ are the final mass eigenstates. As will be discussed in detail in Sec. III, the electroweak precision test (EWPT), including the $\mathrm{T}$ parameter and Z-pole measurements, put a strong constraint on the mixing angle thus the mixing should be very small, which further indicates $c_{\beta}^{2} \ll 1$. Then the value of $\sin \alpha$ can be approximately given by:

$$
\sin \alpha \sim-\frac{2 g_{D} c_{\beta}^{2}}{\sqrt{g^{2}+g^{\prime 2}}} \frac{m_{Z}^{2}}{m_{K}^{2}-m_{Z}^{2}},
$$

where we have kept the leading terms in a $c_{\beta}^{2}$ expansion.

The mass eigenvalues of the gauge bosons are simply:

$$
\begin{gathered}
m_{\tilde{Z}}^{2} \approx m_{Z}^{2}-\sin ^{2} \alpha\left(m_{K}^{2}-m_{Z}^{2}\right)+\mathcal{O}\left(\sin ^{3} \alpha\right) \\
m_{\tilde{K}}^{2} \approx m_{K}^{2}+\frac{4 g_{D}^{2} c_{\beta}^{2}}{g^{2}+g^{\prime 2}} m_{Z}^{2}+\sin ^{2} \alpha\left(m_{K}^{2}-m_{Z}^{2}\right)+\mathcal{O}\left(\sin ^{3} \alpha\right),
\end{gathered}
$$

Due to the mixing between $\tilde{K}$ and $\tilde{Z}$, the coupling of $\tilde{Z}$ to SM particles and also the $\tilde{Z}$ mass will be modified with respect to their SM values. We will carefully discuss it afterwards. At 1-loop level, the kinetic mixing term $\epsilon K_{\mu \nu} B^{\mu \nu}$ can be induced from the fermions which charged under both $U(1)_{Y}$ and $U(1)_{D}$, with $\epsilon \sim g_{D} g^{\prime} /\left(16 \pi^{2}\right)$. Given it is much smaller than the direct mixing $\sin \alpha$ from vev of $\Phi_{1}$, we can neglect this term.

\section{B. Higgs sector}

In this subsection, we will discuss the Higgs sector and get the mass eigenstates of Higgs. First, we write down the general scalar potential which is gauge invariant under $S U(3)_{C} \times S U(2)_{L} \times U(1)_{Y} \times U(1)_{D}$ as follows:

$$
\begin{aligned}
V= & \mu_{1}^{2} \Phi_{1}^{\dagger} \Phi_{1}+\mu_{2}^{2} \Phi_{2}^{\dagger} \Phi_{2}+\mu_{3}^{2} \Phi_{3}^{\dagger} \Phi_{3}+\lambda_{1}\left(\Phi_{1}^{\dagger} \Phi_{1}\right)^{2} \\
& +\lambda_{2}\left(\Phi_{2}^{\dagger} \Phi_{2}\right)^{2}+\lambda_{3}\left(\Phi_{3}^{\dagger} \Phi_{3}\right)^{2}+\lambda_{4}\left(\Phi_{1}^{\dagger} \Phi_{1}\right)\left(\Phi_{2}^{\dagger} \Phi_{2}\right) \\
& +\lambda_{5}\left(\Phi_{1}^{\dagger} \Phi_{1}\right)\left(\Phi_{3}^{\dagger} \Phi_{3}\right)+\lambda_{6}\left(\Phi_{2}^{\dagger} \Phi_{2}\right)\left(\Phi_{3}^{\dagger} \Phi_{3}\right) \\
& +\lambda_{7}\left(\Phi_{1}^{\dagger} \Phi_{2}\right)\left(\Phi_{2}^{\dagger} \Phi_{1}\right)+\mu_{8}\left(\Phi_{1}^{\dagger} \Phi_{2} \Phi_{3}^{*}+\text { H.c. }\right),
\end{aligned}
$$

The minimum condition of $V$ can be always satisfied by requiring the mass terms have the following relationship

$$
\begin{aligned}
& \mu_{1}^{2}=-\left(\lambda_{1} v_{1}^{2}+\frac{\lambda_{4}+\lambda_{7}}{2} v_{2}^{2}+\frac{\lambda_{5}}{2} v_{D}^{2}+\mu_{8} \frac{v_{2} v_{D}}{\sqrt{2} v_{1}}\right), \\
& \mu_{2}^{2}=-\left(\lambda_{2} v_{2}^{2}+\frac{\lambda_{4}+\lambda_{7}}{2} v_{1}^{2}+\frac{\lambda_{6}}{2} v_{D}^{2}+\mu_{8} \frac{v_{1} v_{D}}{\sqrt{2} v_{2}}\right), \\
& \mu_{3}^{2}=-\left(\lambda_{3} v_{D}^{2}+\frac{1}{2}\left(\lambda_{5} v_{1}^{2}+\lambda_{6} v_{2}^{2}\right)+\mu_{8} \frac{v_{1} v_{2}}{\sqrt{2} v_{D}},\right),
\end{aligned}
$$

where the vevs of the Higgs are defined in Eq. (4). Let us start from the charged Higgs mass matrix, which is straightforward to obtain by the second derivative of the potential $V$ :

$$
M_{ \pm}^{2}=-\left(\frac{\lambda_{7}}{2}+\mu_{8} \frac{v_{D}}{\sqrt{2} v_{1} v_{2}}\right)\left(\begin{array}{cc}
v_{2}^{2} & -v_{1} v_{2} \\
-v_{1} v_{2} & v_{1}^{2}
\end{array}\right) .
$$

The mass of the physical charged Higgs is

$$
m_{H^{ \pm}}^{2}=-\frac{\mu_{8} v_{D}}{\sqrt{2} \sin \beta \cos \beta}-\frac{\lambda_{7} v^{2}}{2} .
$$

The two charged Higgs fields $h_{1}^{ \pm}$and $h_{2}^{ \pm}$mix to form the mass eigenstates $H^{ \pm}$and $G^{ \pm}$according to

$$
\left(\begin{array}{l}
h_{1}^{ \pm} \\
h_{2}^{ \pm}
\end{array}\right)=\left(\begin{array}{cc}
\sin \beta & \cos \beta \\
-\cos \beta & \sin \beta
\end{array}\right)\left(\begin{array}{l}
H^{ \pm} \\
G^{ \pm}
\end{array}\right) .
$$

Similarly, we can obtain the mass eigenvalue of physical $C P$-odd Higgs as the trace of the mass matrix: 


$$
M_{\mathrm{odd}}^{2}=-\frac{\mu_{8}}{\sqrt{2}}\left(\begin{array}{ccc}
\frac{v_{2} v_{D}}{v_{1}} & -v_{D} & v_{2} \\
-v_{D} & \frac{v_{1} v_{D}}{v_{2}} & -v_{1} \\
v_{2} & -v_{1} & \frac{v_{1} v_{2}}{v_{D}}
\end{array}\right)
$$

whose value is given by:

$$
m_{A_{0}}^{2}=-\frac{\mu_{8} v_{D}}{\sqrt{2} s_{\beta} c_{\beta}}-\frac{\mu_{8} v^{2} s_{\beta} c_{\beta}}{\sqrt{2} v_{D}},
$$

where we have abbreviated $s_{\beta} \equiv \sin \beta, c_{\beta} \equiv \cos \beta$. The mass mixing matrix is listed in Appendix B. From the masses, we can easily see that in the large $t_{\beta}$ limit, which is required by the small $\tilde{K}, \tilde{Z}$ mixing, the mass scales of the heavy charged Higgs and $C P$-odd Higgs can be as large as $\mathrm{TeV}$ if $\mu_{8}$ is around the electroweak scale. In this limit, both heavy charged Higgs and $C P$-odd Higgs dominantly come from $\Phi_{1}$.

Finally we consider the $C P$-even sector, which involves three physical states. The mass matrix is obtained as follows:

$$
M_{\mathrm{even}}^{2}=\left(\begin{array}{ccc}
2 \lambda_{1} v_{1}^{2}-\frac{\mu_{8} v_{2} v_{D}}{\sqrt{2} v_{1}} & v_{1} v_{2}\left(\lambda_{4}+\lambda_{7}\right)+\frac{\mu_{8} v_{D}}{\sqrt{2}} & \lambda_{5} v_{1} v_{D}+\frac{\mu_{8} v_{2}}{\sqrt{2}} \\
v_{1} v_{2}\left(\lambda_{4}+\lambda_{7}\right)+\frac{\mu_{8} v_{D}}{\sqrt{2}} & 2 \lambda_{2} v_{2}^{2}-\frac{\mu_{8} v_{1} v_{D}}{\sqrt{2} v_{2}} & \lambda_{6} v_{2} v_{D}+\frac{\mu_{8} v_{1}}{\sqrt{2}} \\
\lambda_{5} v_{1} v_{D}+\frac{\mu_{8} v_{2}}{\sqrt{2}} & \lambda_{6} v_{2} v_{D}+\frac{\mu_{8} v_{1}}{\sqrt{2}} & 2 \lambda_{3} v_{D}^{2}-\frac{\mu_{8} v_{1} v_{2}}{\sqrt{2} v_{D}}
\end{array}\right)
$$

As discussed before, in order to decouple the heavy charged Higgs and not induce the large mixing between SM Higgs and the other $C P$-even Higgs, we require that $\mu_{8}$ is roughly of $\mathcal{O}\left(v_{2}\right)$ and $c_{\beta} \ll 1$. In order not to induce large mixing between the SM Higgs $h_{2}$ and the singlet $h_{3}$, we further require that $\lambda_{6}$ is small and of the same order as $c_{\beta}$. Under the above assumption, we can simplify the mass matrix by eliminating the quadratic and linear term of $v_{1}$, except $v_{1} \mu_{8}$ terms, which since $v_{D}$ is of the same order as $v$, are of the same order as $\lambda_{6} v_{2} v_{D}$. This is equivalent to set $\lambda_{1}$, $\lambda_{4}, \lambda_{5}, \lambda_{7}$ to 0 and the $C P$-even mass matrix is now:

$M_{\mathrm{even}}^{2}=\left(\begin{array}{ccc}-\frac{\mu_{8} v_{2} v_{D}}{\sqrt{2} v_{1}} & \frac{\mu_{8} v_{D}}{\sqrt{2}} & \frac{\mu_{8} v_{2}}{\sqrt{2}} \\ * & 2 \lambda_{2} v_{2}^{2}-\frac{\mu_{8} v_{1} v_{D}}{\sqrt{2} v_{2}} & \lambda_{6} v_{2} v_{D}+\frac{\mu_{8} v_{1}}{\sqrt{2}} \\ * & * & 2 \lambda_{3} v_{D}^{2}-\frac{\mu_{8} v_{1} v_{2}}{\sqrt{2} v_{D}}\end{array}\right)$

The mass eigenvalues at leading order in $\cot \beta$ and $\lambda_{6}$ are simply as following:

$$
\begin{aligned}
& m_{H_{1}^{0}}^{2} \simeq-\frac{\mu_{8} v_{D} \tan \beta}{\sqrt{2}} \simeq m_{A^{0}}^{2}, \\
& m_{H_{2}^{0}}^{2} \simeq 2 \lambda_{2} v_{2}^{2}, \\
& m_{H_{3}^{0}}^{2} \simeq 2 \lambda_{3} v_{D}^{2} .
\end{aligned}
$$

The unitary mixing matrix is define as:

$$
\left(\begin{array}{c}
h_{1}^{0} \\
h_{2}^{0} \\
h_{3}^{0}
\end{array}\right)=\left(\begin{array}{lll}
U_{11} & U_{12} & U_{13} \\
U_{21} & U_{22} & U_{23} \\
U_{31} & U_{32} & U_{33}
\end{array}\right)\left(\begin{array}{c}
H_{1}^{0} \\
H_{2}^{0} \\
H_{3}^{0}
\end{array}\right)
$$

where $h(H)$ denote flavor (mass) eigenstates respectively. The entries can be obtained at the leading order in $\cot \beta$ :

$$
\begin{aligned}
& U_{11} \sim U_{22} \sim U_{33} \sim 1+\mathcal{O}\left(\cot ^{2} \beta\right), \\
& U_{12} \sim-U_{21} \simeq \cot \beta, \\
& U_{13} \sim-U_{31} \simeq \cot \beta \frac{v_{2}}{v_{D}}, \\
& U_{23} \sim-U_{32} \sim \mathcal{O}(\cot \beta),
\end{aligned}
$$

where the expression of $U_{23}$ proceeds from a combination of terms proportional to $\cot \beta$ and $\lambda_{6}$, and we set it as a free parameter. The more detailed expressions for the mass of $C P$-even Higgs and mixing matrix $U$ are given in the Appendix A. We can easily see that, in the decoupling limit, the modifications to SM Higgs couplings with massive gauge bosons and the fermions arise at second order in $\cot \beta$, which are therefore at the percent level in our scenario since $\cot \beta \sim 0.1$.

\section{Fermion sector}

Let's now turn to mixing in the fermion sector, where we especially focus on the $b$ and $c$ quarks. As explained above, the masses of the $b$ and $c$ quarks come from the mixing with heavy vector like fermions $\psi_{b, c}$. We first consider the mixing between $\psi_{b}$ and $b$. The $2 \times 2$ mass matrix in $\left(\psi_{b}, b\right)$ basis simply reads:

$$
M_{b}=\left(\begin{array}{cc}
m_{b, \psi} & \frac{y_{3 b, \psi} v_{D}}{\sqrt{2}} \\
\frac{y_{2 b, \psi} v_{2}}{\sqrt{2}} & 0
\end{array}\right) \equiv\left(\begin{array}{cc}
m_{b, \psi} & m_{12}^{b} \\
m_{21}^{b} & 0
\end{array}\right),
$$

where we simply treat the off-diagonal terms as small variables $m_{12}^{b} \ll m_{b, \psi}$. It is straightforward to diagonalize 
the mass matrix by the orthogonal rotation of the left-handed and right-handed quark fields:

$$
\begin{aligned}
& \left(\begin{array}{c}
\psi_{b, L} \\
b_{L}
\end{array}\right)=\left(\begin{array}{cc}
c_{b, L} & s_{b, L} \\
-s_{b, L} & c_{b, L}
\end{array}\right)\left(\begin{array}{c}
\tilde{\psi}_{b, L} \\
\tilde{b}_{L}
\end{array}\right) \\
& \left(\begin{array}{c}
\psi_{b, R} \\
b_{R}
\end{array}\right)=\left(\begin{array}{cc}
c_{b, R} & s_{b, R} \\
-s_{b, R} & c_{b, R}
\end{array}\right)\left(\begin{array}{c}
\tilde{\psi}_{b, R} \\
\tilde{b}_{R}
\end{array}\right),
\end{aligned}
$$

where the mixing angles are approximately given by:

$$
s_{b, L} \sim-\frac{m_{21}^{b}}{m_{b, \psi}}, \quad s_{b, R} \sim-\frac{m_{12}^{b}}{m_{b, \psi}},
$$

and the mass eigenvalues are

$$
m_{\tilde{\psi}_{b}} \simeq m_{b, \psi}, \quad m_{\tilde{b}} \simeq-\frac{m_{12}^{b} m_{21}^{b}}{m_{b, \psi}} \simeq-s_{b, L} s_{b, R} m_{b, \psi},
$$

where the mass formula for the bottom quark is similar to the partial compositeness scenario [12]. The same analysis applies to the charm quark except the parameters are in the charm sector. The mass formula and the mixing angle are given by:

$$
\begin{array}{ll}
m_{\tilde{\psi}_{c}} & \simeq m_{c, \psi}, \quad m_{\tilde{c}} \simeq-\frac{m_{12}^{c} m_{12}^{c}}{m_{c, \psi}} \simeq-s_{c, L} s_{c, R} m_{c, \psi}, \\
s_{c, L} & \sim-\frac{m_{21}^{c}}{m_{c, \psi}}, \quad s_{c, R} \sim-\frac{m_{12}^{c}}{m_{b, \psi}} .
\end{array}
$$

We now consider the mass eigenstates of $\chi_{1,2}$. The Dirac mass term for $\chi_{2}$ is simply:

$$
m_{\chi_{2}}=\frac{y_{\chi_{2}} v_{D}}{\sqrt{2}}
$$

without any mixing with SM particles and this will be our dark matter candidate. At current stage, we assume the elastic DM scenario that Majorana mass $M_{m}=0$, which can be originated from a global continuous symmetry for $\chi_{2}$. We will come back to Majorana DM later. There is a mixing between $\chi_{1}$ and $\tau$ induced by the Dirac mass $m_{\tau 1}$, which we assume to be tiny. So the mass eigenvalues at leading order are simply:

$$
m_{\tilde{\chi}_{1}} \simeq \frac{y_{\chi_{1}} v_{D}}{\sqrt{2}}, \quad m_{\tilde{\tau}} \simeq \frac{y_{\tau} v_{2}}{\sqrt{2}}
$$

At the linear order in $m_{\tau 1} / m_{\chi_{1}}$, only the right-handed part mix with each other:

$$
\left(\begin{array}{c}
\chi_{1, R} \\
\tau_{R}
\end{array}\right)=\left(\begin{array}{cc}
c_{\tau, R} & s_{\tau, R} \\
-s_{\tau, R} & c_{\tau, R}
\end{array}\right)\left(\begin{array}{c}
\tilde{\chi}_{1, R} \\
\tilde{\tau}_{R}
\end{array}\right)
$$

where the mixing angle are

$$
s_{\tau, R} \simeq-\frac{m_{\tau_{1}}}{m_{\tilde{\chi}_{1}}}, \quad s_{\tau, L} \simeq-\frac{m_{\tau_{1}}}{m_{\tilde{\chi}_{1}}} \frac{m_{\tilde{\tau}}}{m_{\tilde{\chi}_{1}}}
$$

and we see clearly $s_{\tau, L} \ll s_{\tau, R}$ and can be neglected.

The relevance of $s_{\tau, R}$ mixing is to let the $\chi_{1}$ decay, so in principle we can make it as small as we want unless the lifetime of $\chi_{1}$ is long enough to have cosmological problems. For example, if we make it as small as $10^{-4}$, it will not affect the SM $\tau$ interactions in any significant way and $\chi_{1}$ will have a decay width $\sim \alpha_{e m} m_{\chi_{1}} s_{\tau, R}^{2} \sim 10 \mathrm{eV}$, implying that it will still decay promptly at the LHC.

\section{Gauge bosons interactions with fermions}

In this section, we will review the interactions between the fermions and the gauge bosons. Let us emphasize again that the gauge eigenstates of gauge bosons (e.g. $Z$ and $K$ ) are denoted without tildes, while the mass eigenstates (e.g. $\tilde{Z}$ and $\tilde{K}$ ) are denoted with tildes. For the gauge bosons $W^{ \pm}$ and photon $A$, no further mixing are induced by $U(1)_{D}$ and thus they are the same as in SM. First, we notice that in the gauge basis, the interaction Lagrangian in the quark sector reads:

$$
\begin{aligned}
\mathcal{L}_{\text {int }}^{I}= & \frac{g}{\sqrt{2}} W_{\mu}^{+} \bar{t}_{L} \gamma^{\mu} b_{L}+\frac{g}{2 c_{w}} Z_{\mu}\left(-\bar{b}_{L} \gamma^{\mu} b_{L}+\bar{c}_{L} \gamma^{\mu} c_{L}\right) \\
& +g_{D} K_{\mu}\left(\bar{b}_{R} \gamma^{\mu} b_{R}-\bar{c}_{R} \gamma^{\mu} c_{R}\right) \\
& +\frac{g s_{w}^{2}}{3 c_{w}} Z_{\mu}\left(\bar{b} \gamma^{\mu} b+\bar{\psi}_{b} \gamma^{\mu} \psi_{b}\right) \\
& -\frac{2 g s_{w}^{2}}{3 c_{w}} Z_{\mu}\left(\bar{c} \gamma^{\mu} c+\bar{\psi}_{c} \gamma^{\mu} \psi_{c}\right)
\end{aligned}
$$

where we neglect the photon couplings as it is only determined by the electric charge of the fermions, not changing the couplings of $K$ and $Z$. To determine the couplings of $Z$, we separate the electric-charge $(Q)$ part and the weak isospin part $T^{3}$. Because the electromagnetic gauge symmetry is unbroken, only particles with the same electric-charge can mix with each other after EWSB, making the $Q$ part of the $Z$ couplings flavor diagonal. Then the only flavor off-diagonal $Z$ coupling comes from the $T^{3}$ contribution, namely

$$
\frac{g}{2 c_{w}} Z_{\mu}\left(-\bar{b}_{L} \gamma^{\mu} b_{L}+\bar{c}_{L} \gamma^{\mu} c_{L}\right)
$$


which are purely left-handed. In contrast, the $K$ couplings are purely right-handed. Now it is easy to obtain the gauge boson couplings in the mass eigenstate by performing the orthogonal rotation to the gauge bosons and the fermions. The results for the SM charge gauge bosons read:

$$
\mathcal{L}_{\text {int }}^{W}=\frac{g}{\sqrt{2}} W_{\mu}^{+} \bar{t}_{L} \gamma^{\mu}\left(c_{b, L} \tilde{b}_{L}-s_{b, L} \tilde{\psi}_{b, L}\right)+\text { H.c. }
$$

and for the neutral $\tilde{Z}_{\mu}$ state the interactions read

$$
\begin{aligned}
\mathcal{L}_{\text {int }}^{Z}= & \tilde{Z}_{\mu}\left[\overline{\tilde{b}}_{L} \gamma^{\mu} \tilde{b}_{L} \frac{g \cos \alpha}{c_{w}}\left(\frac{s_{w}^{2}}{3}-\frac{1}{2} c_{b, L}^{2}\right)+\overline{\tilde{b}}_{R} \gamma^{\mu} \tilde{b}_{R}\left(\frac{g s_{w}^{2}}{3 c_{w}} \cos \alpha-g_{D} \sin \alpha c_{b, R}^{2}\right)\right] \\
& +\tilde{Z}_{\mu}\left[\overline{\tilde{c}}_{L} \gamma^{\mu} \tilde{c}_{L} \frac{g \cos \alpha}{c_{w}}\left(-\frac{2 s_{w}^{2}}{3}+\frac{1}{2} c_{c, L}^{2}\right)+\overline{\tilde{c}}_{R} \gamma^{\mu} \tilde{c}_{R}\left(-\frac{2 g s_{w}^{2}}{3 c_{w}} \cos \alpha+g_{D} \sin \alpha c_{c, R}^{2}\right)\right] \\
& +\frac{g \cos \alpha s_{w}^{2}}{3 c_{w}} \tilde{Z}_{\mu}\left\{\left[c_{b, L}^{2} \overline{\tilde{\psi}}_{b, L} \gamma^{\mu} \tilde{\psi}_{b, L}+(L \leftrightarrow R)\right]-2\left[c_{c, L}^{2} \overline{\tilde{\psi}}_{c, L} \gamma^{\mu} \tilde{\psi}_{c, L}+(L \leftrightarrow R)\right]\right\} \\
& +\tilde{Z}_{\mu}\left[\left(\frac{g \cos \alpha c_{b, L} s_{b, L}}{2 c_{w}} \overline{\tilde{\psi}}_{b, L} \gamma^{\mu} \tilde{b}_{L}+g_{D} \sin \alpha c_{b, R} s_{b, R} \overline{\tilde{\psi}}_{b, R} \gamma^{\mu} \tilde{b}_{R}+\text { H.c. }\right)-(b \leftrightarrow c)\right]
\end{aligned}
$$

where the mixing angles are defined in the previous two sections. We can clearly see that the modifications to the $Z \bar{b}_{R} b_{R}$ and the $Z \bar{c}_{R} c_{R}$ couplings come at linear order in $\sin \alpha$ and are of opposite sign, while for the left-handed couplings, they arise from the normalization of the quark fields starting at the square order of the mixing parameters $\sin ^{2} \alpha, s_{c, L}^{2}, s_{b, L}^{2}$. As we will see later, a small modification to the left-handed bottom and charm $Z$ boson couplings is necessary in order to satisfy the total $b, c$ hadronic cross section measurements on the Z-pole.

For the $U(1)_{D}$ gauge boson interactions at lowest order, we have:

$$
\begin{aligned}
\mathcal{L}_{\text {int }}^{K}= & \frac{g \sin \alpha}{c_{w}} \tilde{K}_{\mu} J_{Z, q}^{\mu}+\tilde{K}_{\mu}\left[\overline{\tilde{b}}_{L} \gamma^{\mu} b_{L} \frac{g \sin \alpha}{c_{w}}\left(\frac{s_{w}^{2}}{3}-\frac{1}{2} c_{b, L}^{2}\right)+\overline{\tilde{b}}_{R} \gamma^{\mu} \tilde{b}_{R}\left(\frac{g s_{w}^{2}}{3 c_{w}} \sin \alpha+g_{D} \cos \alpha c_{b, R}^{2}\right)\right] \\
& +\tilde{K}_{\mu}\left[\overline{\tilde{c}}_{L} \gamma^{\mu} c_{L} \frac{g \sin \alpha}{c_{w}}\left(-\frac{2 s_{w}^{2}}{3}+\frac{1}{2} c_{c, L}^{2}\right)+\overline{\tilde{c}}_{R} \gamma^{\mu} \tilde{c}_{R}\left(-\frac{2 g s_{w}^{2}}{3 c_{w}} \sin \alpha-g_{D} \cos \alpha c_{c, R}^{2}\right)\right] \\
& +\tilde{K}_{\mu}\left(\overline{\tilde{\psi}}_{b, L} \gamma^{\mu} \tilde{b}_{L} \frac{g}{2 c_{w}} \sin \alpha c_{b, L} s_{b, L}-\overline{\tilde{\psi}}_{b, R} \gamma^{\mu} \tilde{b}_{R} g_{D} \cos \alpha c_{b, R} s_{b, R}\right) \\
& -\tilde{K}_{\mu}\left(\overline{\tilde{\psi}}_{c, L} \gamma^{\mu} \tilde{c}_{L} \frac{g}{2 c_{w}} \sin \alpha c_{c, L} s_{c, L}-\overline{\tilde{\psi}}_{c, R} \gamma^{\mu} \tilde{c}_{R} g_{D} \cos \alpha c_{c, R} s_{c, R}\right)
\end{aligned}
$$

where $J_{Z, q}^{\mu}$ is the SM quark neutral currents except the bottom and charm quarks:

$$
J_{Z, q}^{\mu}=\sum_{q \neq b, c}\left(T^{3}-Q s_{w}^{2}\right) \bar{q} \gamma^{\mu} q
$$

We can see that $\tilde{K}_{\mu}$ mainly couples to the SM right-handed bottom and charm quarks with gauge coupling $g_{D}$ and couples universally to other quarks and leptons through its small mixing with $Z$ boson. We finally comment that due to the existence of a Dirac mass for the vector-like quark $\psi_{b}$ and $\psi_{c}$, one can lift these vectorlike fermion masses $(\gtrsim 1 \mathrm{TeV})$ to decouple $\psi_{b}$ and $\psi_{c}$ from LHC physics, while choose appropriate mixing angles to give the right mass to the $b$ and $c$ quarks.

Next we consider the gauge boson interactions in the lepton sector including $\tau$ and $\chi_{1,2}$. The interaction Lagrangian in gauge basis reads:

$$
\mathcal{L}_{\chi}=-e A_{\mu}\left(\bar{\chi}_{1} \gamma^{\mu} \chi_{1}+\bar{\tau} \gamma^{\mu} \tau\right)+Z_{\mu}\left(\frac{g s_{w}^{2}}{c_{w}}\left(\bar{\chi}_{1} \gamma^{\mu} \chi_{1}+\bar{\tau} \gamma^{\mu} \tau\right)-\frac{g}{2 c_{w}} \bar{\tau}_{L} \gamma^{\mu} \tau_{L}\right)+K_{\mu} g_{D}\left(\bar{\chi}_{1, R} \gamma^{\mu} \chi_{1, R}-\bar{\chi}_{2, R} \gamma^{\mu} \chi_{2, R}\right)
$$

In the mass eigenstate basis, the Lagrangian at leading order mixing is, 


$$
\begin{aligned}
\mathcal{L}_{\chi} \simeq & -e A_{\mu}\left(\overline{\tilde{\chi}}_{1} \gamma^{\mu} \tilde{\chi}_{1}+\overline{\tilde{\tau}} \gamma^{\mu} \tilde{\tau}\right)+\frac{g \sin \alpha}{c_{w}} \tilde{K}_{\mu} J_{Z, \ell}^{\mu}+\tilde{Z}_{\mu}\left(\cos \alpha\left(\frac{g s_{w}^{2}}{c_{w}}\left(\overline{\tilde{\chi}}_{1} \gamma^{\mu} \tilde{\chi}_{1}+\overline{\tilde{\tau}} \gamma^{\mu} \tilde{\tau}\right)-\frac{g}{2 c_{w}} \overline{\tilde{\tau}}_{L} \gamma^{\mu} \tilde{\tau}_{L}\right)\right. \\
& \left.-g_{D} \sin \alpha\left(c_{\tau, R}^{2} \overline{\tilde{\chi}}_{1, R} \gamma^{\mu} \tilde{\chi}_{1, R}-\bar{\chi}_{2, R} \gamma^{\mu} \chi_{2, R}\right)\right)+\tilde{K}_{\mu}\left(\sin \alpha\left(\frac{g s_{w}^{2}}{c_{w}}\left(\overline{\tilde{\chi}}_{1} \gamma^{\mu} \tilde{\chi}_{1}+\overline{\tilde{\tau}} \gamma^{\mu} \tilde{\tau}\right)-\frac{g}{2 c_{w}} \overline{\tilde{\tau}}_{L} \gamma^{\mu} \tilde{\tau}_{L}\right)\right. \\
& \left.+g_{D} \cos \alpha\left(c_{\tau, R}^{2} \overline{\tilde{\chi}}_{1, R} \gamma^{\mu} \tilde{\chi}_{1, R}-\bar{\chi}_{2, R} \gamma^{\mu} \chi_{2, R}\right)\right)+g_{D}\left(\cos \alpha \tilde{K}_{\mu}-\sin \alpha \tilde{Z}_{\mu}\right) c_{\tau, R} s_{\tau, R}\left(\overline{\tilde{\tilde{\chi}}}_{1, R} \gamma^{\mu} \tilde{\tau}_{R}+\text { H.c. }\right) .
\end{aligned}
$$

where $J_{Z, \ell}^{\mu}$ is the SM lepton neutral currents except the $\tau$ :

$$
J_{Z, \ell}^{\mu}=\sum_{\ell \neq \tau}\left(T^{3}-Q s_{w}^{2}\right) \bar{\ell} \gamma^{\mu} \ell
$$

As explained in previous subsection, $s_{\tau, R}$ can be chosen to be very small to make $\chi_{1}$ decay promptly at LHC while not affecting the early cosmology. We note that $\chi_{1}$ has mass around $\sim v_{D}$, thus is relevant for LHC physics. Later we will show that due to its coupling only to hypercharge, it is not constrained by current LHC limits.

\section{E. Higgs interaction with fermions and gauge bosons}

After we consider the mass eigenstates of Higgs and fermions, we can have the following interactions:

$$
\begin{aligned}
\mathcal{L}_{y u k}^{I}= & -\left(\frac{m_{t}}{v s_{\beta}} \overline{\tilde{t}}_{L} \tilde{t}_{R}+\frac{m_{s}}{v s_{\beta}} \overline{\tilde{s}}_{L} \tilde{s}_{R}\right)\left(-c t_{\beta} H_{1}^{0}+U_{22} H_{2}^{0}+U_{23} H_{3}^{0}\right)-\frac{m_{\tilde{b}}}{s_{\beta} v} \overline{\tilde{b}}_{L} \tilde{b}_{R}\left(-\left(c t_{\beta} c_{b, L}+\frac{c_{\beta} s_{\beta} v^{2}}{v_{D}^{2}} c_{b, R}\right) H_{1}^{0}\right. \\
& \left.+c_{b, L} U_{22} H_{2}^{0}+\frac{s_{\beta} v}{v_{D}} c_{b, R} U_{33} H_{3}^{0}\right)-\frac{m_{\tilde{c}}}{s_{\beta} v} \overline{\tilde{c}}_{L} \tilde{c}_{R}\left(-\left(c t_{\beta} c_{c, L}+\frac{c_{\beta} s_{\beta} v^{2}}{v_{D}^{2}} c_{c, R}\right) H_{1}^{0}+c_{c, L} U_{22} H_{2}^{0}+\frac{s_{\beta} v}{v_{D}} c_{c, R} U_{33} H_{3}^{0}\right) \\
& -\left(\frac{m_{\chi_{2}}}{v_{D}} \bar{\chi}_{2, L} \chi_{2, R}+\frac{m_{\tilde{\chi}_{1}} c_{\tau, R}}{v_{D}} \overline{\tilde{\chi}}_{1, L} \tilde{\chi}_{1, R}+\frac{m_{\tilde{\chi}_{1}} s_{\tau, R}}{v_{D}} \overline{\tilde{\chi}}_{1, L} \tilde{\tau}_{R}\right)\left(-\frac{v}{v_{D}} c_{\beta} H_{1}^{0}-U_{23} H_{2}^{0}+U_{33} H_{3}^{0}\right) \\
& -\left(\frac{m_{\tilde{\tau}} c_{\tau, R}}{s_{\beta} v} \overline{\tilde{\tau}}_{L} \tilde{\tau}_{R}-\frac{m_{\tilde{\tau}} s_{\tau, R}}{s_{\beta} v} \overline{\tilde{\tau}}_{L} \tilde{\chi}_{1, R}\right)\left(-c t_{\beta} H_{1}^{0}+U_{22} H_{2}^{0}+U_{23} H_{3}^{0}\right),
\end{aligned}
$$

where we have abbreviated $c_{\beta} \equiv \cos \beta, c t_{\beta} \equiv \cot \beta, \cdots$ etc. and substituted the leading values for $U_{12}$ and $U_{13}$ in Eq. (29). Note that we have only kept the leading term in the $H_{2}^{0}\left(H_{3}^{0}\right) b \bar{b}(c \bar{c})$ couplings in the limit $c_{\beta} \ll 1$. Since $s_{\beta} \simeq 1$, the SM-like Higgs boson $H_{2}^{0}$ will couple to SM fermions the same as the standard nodel except from $\mathcal{O}\left(c_{\beta}^{2}\right)$ corrections, which are at the percent level in our model. This implies that this model cannot be tested through Higgs fermion coupling measurements at the LHC and hence we shall not discuss these constraints anymore. We also see that the $H_{3}^{0}$ is $\Phi_{3}$-like and coupled to bottom and charm quark proportional to their mass as discussed before. Note that it also couples to top quark through its mixing with $h_{2}^{0}$, which maybe relevant due to the large top Yukawa coupling and the mixing size of order $c t_{\beta}$.

In the following, we will consider the mass hierarchy $m_{H_{2}^{0}}, m_{H_{3}^{0}} \lesssim m_{\tilde{\chi}_{1}} \ll m_{H_{1}^{0}} \ll m_{\tilde{\psi}_{b, c}}$. Hence, the heavy charged lepton $\chi_{1}$ can decay to scalars plus $\tau$ leptons, where the leading channel is $\tau H_{3}^{0}$ which is only suppressed by $s_{\tau, R}^{2}$, while the channel $\tau H_{2}^{0}$ is further suppressed by tau mass. Given Eq. (46), the other dominant decay channel for $\chi_{1}$ is $\tau \tilde{K}$ which is also of order $s_{\tau, R}^{2}$. Therefore, $\tilde{\chi}_{1}$ decays into $\tau(\bar{b} b)$ and $\tau(\bar{c} c)$, which could be a new signature to look for at LHC depending on the production cross section of $\chi_{1}$.

For completeness, we list the leading interaction between $\psi$ and $c, b$, and neglect the quadratic terms like $\mathcal{O}\left(s_{b, c}^{2}, s_{b, c} c_{\beta}\right)$,

$$
\begin{aligned}
-\mathcal{L}_{y u k}^{I} \supset & s_{b, L} m_{\tilde{\psi}_{b}} \frac{U_{22} H_{2}^{0}}{v_{2}} c_{b, L} c_{b, R} \overline{\tilde{b}}_{L} \tilde{\psi}_{b, R} \\
& +s_{b, R} m_{\tilde{\psi}_{b}} \frac{U_{33} H_{3}^{0}}{v_{D}} c_{b, L} c_{b, R} \overline{\tilde{\psi}}_{b, L} \tilde{b}_{R}+\text { H.c. } \\
& +s_{c, L} m_{\tilde{\psi}_{c}} \frac{U_{22} H_{2}^{0}}{v_{2}} c_{c, L} c_{c, R} \overline{\tilde{c}}_{L} \tilde{\psi}_{c, R} \\
& +s_{c, R} m_{\tilde{\psi}_{c}} \frac{U_{33} H_{3}^{0}}{v_{D}} c_{c, L} c_{c, R} \overline{\tilde{\psi}}_{c, L} \tilde{c}_{R}+\text { H.c.. }
\end{aligned}
$$

Note that the couplings to diagonal heavy quark $\tilde{\tilde{\psi}} \tilde{\psi}$ are neglected at $\mathcal{O}\left(s_{b, c}^{2}\right)$. The vector-like quark $\tilde{\psi}_{b, c}$ can decay into $\tilde{b}, \tilde{c}$ quarks plus $\tilde{Z}, \tilde{K}$ and scalars. The decay width to 
$\tilde{Z}, \tilde{K}, H_{2}^{0}, H_{3}^{0}$ are proportional to $s_{q, L}^{2}, s_{q, R}^{2}, s_{q, L}^{2} m_{\tilde{\psi}_{q}}^{2} / v_{2}^{2}$, $s_{q, R}^{2} m_{\tilde{\psi}_{q}}^{2} / v_{D}^{2}$. Given that the Dirac mass of $\psi$ is much larger than $v_{2} \sim v_{D}$, the dominant decay channels for $\psi_{b, c}$ are $b, c$ plus scalars. Since one can give a large enough Dirac mass for $\tilde{\psi}_{b, c}$ to evade the collider constraints, we will not further discuss their search at LHC.

Next, we consider the Yukawa interaction with charged Higgs $H^{ \pm}$and $C P$-odd Higgs $A^{0}$. The Lagrangian for the charged Higgs in the mass eigenstates reads:

$$
\begin{aligned}
\mathcal{L}_{\text {int }}^{H^{ \pm}}= & +\frac{\sqrt{2} m_{b}}{t_{\beta} v} \bar{t}_{L} H^{+}\left(\tilde{b}_{R}+\frac{c_{b, R}}{s_{b, R}} \tilde{\psi}_{b, R}\right) \\
& -\frac{\sqrt{2} m_{t}}{t_{\beta} v}\left(c_{b, L} \overline{\tilde{b}}_{L}-s_{b, L} \overline{\tilde{\psi}}_{b, L}\right) H^{-} t_{R}+\text { H.c. } \\
& +\frac{\sqrt{2} m_{s}}{t_{\beta} v}\left(c_{c, L} \overline{\tilde{c}}_{L}-s_{c, L} \overline{\tilde{\psi}}_{c, L}\right) H^{+} s_{R} \\
& -\frac{\sqrt{2} m_{c}}{t_{\beta} v} \bar{s}_{L} H^{-}\left(\tilde{c}_{R}+\frac{c_{c, R}}{s_{c, R}} \tilde{\psi}_{c, R}\right)+\text { H.c. }
\end{aligned}
$$

The fermion interaction with $A^{0}$ is given in the Appendix B. As discussed before, $H^{ \pm}$and $A^{0}$ can be made as heavy as $\mathrm{TeV}$, therefore we are not going to discuss them further.

We finally list the interactions between one $C P$-even scalar and two gauge bosons, which maybe relevant for the LHC phenomenology. The Lagrangian in the gauge basis at leading $c_{\beta}$ order is

$$
\begin{aligned}
\mathcal{L}_{\phi V V}= & \frac{2 m_{W}^{2}}{v} W^{+\mu} W_{\mu}^{-}\left(c_{\beta} h_{1}^{0}+s_{\beta} h_{2}^{0}\right) \\
& +\frac{m_{Z}^{2}}{v}\left[c_{\beta} h_{1}^{0}\left(Z_{\mu}+\frac{2 g_{D} c_{w}}{g} K_{\mu}\right)^{2}+s_{\beta} h_{2}^{0} Z_{\mu} Z^{\mu}\right] \\
& +h_{3}^{0} \frac{m_{K}^{2}}{v_{D}} K_{\mu}^{2},
\end{aligned}
$$

where the couplings of gauge bosons with the scalars are determined by the scalars' contributions to the mass of the gauge bosons. The Lagrangian for the mass eigenstates are

$$
\begin{aligned}
\mathcal{L}_{\phi V V} \simeq & \frac{2 m_{W}^{2}}{v} W^{\mu+} W_{\mu}^{-}\left(c_{\beta}\left(U_{11}-1\right) H_{1}^{0}+s_{\beta} U_{22} H_{2}^{0}+s_{\beta} U_{23} H_{3}^{0}\right)+\frac{m_{Z}^{2}}{v} \tilde{Z}^{\mu} \tilde{Z}_{\mu}\left(c_{\beta}\left(\left(U_{11}-1\right) c_{\alpha}^{2}+s_{2 \alpha} \frac{g_{D} v}{m_{Z}}\right) H_{1}^{0}+c_{\alpha}^{2} s_{\beta} U_{22} H_{2}^{0}\right. \\
& \left.+c_{\alpha}^{2} s_{\beta} U_{23} H_{3}^{0}\right)+\tilde{K}_{\mu} \tilde{K}^{\mu}\left(\left(c_{\alpha}^{2} c_{\beta} g_{D}^{2} v\left(U_{11}-1\right)-c_{\beta} s_{2 \alpha} g_{D} m_{Z} U_{11}\right) H_{1}^{0}-c_{\alpha}^{2} \frac{m_{K}^{2}}{v_{D}} U_{23} H_{2}^{0}+c_{\alpha}^{2} \frac{m_{K}^{2}}{v_{D}} U_{33} H_{3}^{0}\right) \\
& +\tilde{Z}_{\mu} \tilde{K}^{\mu}\left(-2 c_{\alpha}^{2} c_{\beta} g_{D} m_{Z} U_{11} H_{1}^{0}+\frac{s_{2 \alpha} s_{\beta} m_{Z}^{2}}{v} U_{22} H_{2}^{0}-s_{2 \alpha} \frac{m_{K}^{2}}{v_{D}} U_{33} H_{3}^{0}\right)
\end{aligned}
$$

where we have kept leading terms in $c_{\beta}$ and $s_{\alpha}$ for $H_{1,2,3}^{0}$ term respectively. We can see that $H_{2}^{0}$ couplings to gauge bosons are modified at the percent level $\sim c_{\beta}^{2}$, which is consistent with the present precision at the LHC. The $H_{1}^{0}$ couplings are further suppressed at quadratic or cubed order, $\mathcal{O}\left(c_{\beta}^{3}, c_{\beta} s_{\alpha}, s_{\alpha}^{2}\right)$, though linearly suppressed by $c_{\beta}$ for $\tilde{Z} \tilde{K}$ coupling, while $H_{2}^{0}, H_{3}^{0}$ are at most suppressed by linear $c_{\beta}$ or $s_{\alpha}$. This fact reveals that it is much more difficult to search for $H_{1}^{0}$ at the LHC.

For the $H_{3}^{0}$, it couples largely to the $\tilde{K}$ gauge boson as it is the main source of $\tilde{K}$ gauge boson mass. As a result, if $m_{\chi_{1}, \chi_{2}}>m_{H_{3}^{0}} / 2$, it will dominantly decay into $\tilde{K}$ pair if this decay channel is kinematically open. It can also decay into $\tilde{b} \bar{b}, \tilde{c} \overline{\tilde{c}}$ pairs which may be dominant if the $\tilde{K}$ decay channel is closed. It could decay into other SM fermions pair but will be suppressed by the mixing between $H_{2}^{0}$ and $H_{3}^{0}$. Concerning its production at the LHC, we expect that it is mainly produced through $g g$ fusion due to top and bottom loops. If $U_{23}$ is of order $c t_{\beta}$, top loop will dominate. In this case, its production cross section at the LHC will be suppressed by $c t_{\beta}^{2} \simeq 0.01$ compared with a SM-like Higgs boson of the same mass, namely around $\sigma_{13 \mathrm{TeV}}\left(p p \rightarrow H_{3}^{0}\right) \simeq 0.44 \mathrm{pb}$ and $\sigma_{13 \mathrm{TeV}}\left(p p \rightarrow H_{3}^{0} j j\right) \simeq$ $0.037 \mathrm{pb}$ for $m_{H_{3}^{0}}=125 \mathrm{GeV}$. These cross sections are too small to discriminate the $H_{3}^{0}$ production from the multijet QCD background. If $m_{\chi_{2}}<m_{H_{3}^{0}} / 2$, the most promising scenario for searching $H_{3}^{0}$ is $H_{3}^{0} j j$ production, following by the nearly $100 \%$ invisible decay to $\chi_{2} \bar{\chi}_{2}$, if $m_{\chi_{1}}, m_{\tilde{K}}>$ $m_{H_{3}^{0}} / 2$. Comparing to the cross section of $\sigma(j j(Z \rightarrow \nu \bar{\nu})) \sim$ $10^{3} \mathrm{pb}, H_{3}^{0}$ production is still hard to probe at the LHC.

\section{ELECTROWEAK PRECISION MEASUREMENTS}

The main motivation behind this model is the observed $3 \sigma$ deviation of the bottom-quark forward-backward asymmetry $A_{\mathrm{FB}}^{b}$ measured at the LEP experiment at CERN. It is well known that this asymmetry may be modified by varying the right-handed bottom coupling to the Z-boson $[5,10,14-18]$. In general, the modification of the couplings produces other effects that have relevant implications on the precision electroweak observables, which should be considered simultaneously. In fact, the strongest constraints on this model come precisely from the Electroweak precision 
measurements [3,19-22] including the $\mathrm{T}$ parameter and the $Z$-pole observables. In our setup, the mixing between $\tilde{K}$ and $\tilde{Z}$ will induce the custodial symmetry breaking, which modifies the $\tilde{Z}$ mass without changing the mass of the $W$ boson. The corresponding contribution to the T-parameter is given by:

$$
\hat{\alpha}\left(m_{Z}\right) T=-\frac{\Delta m_{Z}^{2}}{m_{Z}^{2}} \sim \sin ^{2} \alpha \frac{m_{K}^{2}-m_{Z}^{2}}{m_{Z}^{2}},
$$

where $\hat{\alpha}\left(m_{Z}\right)$ is the value of the fine-structure constant evaluated on the $Z$-pole, whose value is $[23,24]$ :

$$
\hat{\alpha}\left(m_{Z}\right)=\frac{1}{127.95} .
$$

The modification of the T-parameter has the same sign as $m_{K}^{2}-m_{Z}^{2}$. From the T-parameter measurement $T=$ $0.08 \pm 0.12$ [25], we can obtain the $95 \%$ bound on the modification of the $Z$ mass:

$$
\sin ^{2} \alpha \frac{m_{K}^{2}-m_{Z}^{2}}{m_{Z}^{2}} \in[-0.00121,0.00246]
$$

which can translated into the bound on the mixing angle $\sin \alpha$ for given mass of the $\tilde{K}$ gauge boson.

Next, we consider the $Z$-pole measurements, including not only $A_{\mathrm{FB}}^{b}$ but also the total width of the $Z$ boson $\Gamma_{\text {tot }}$, the heavy flavor quarks (bottom and charm quark) production ratio $R_{b, c}$, lepton production ratio $R_{l}$, and the forwardbackward asymmetry of the charm quarks $A_{F B}^{c}$. They can be roughly written in terms of the left-handed and righthanded $Z$-couplings as:

$$
\begin{aligned}
R_{b, c} & \equiv \frac{\Gamma(\tilde{Z} \rightarrow \tilde{b} \overline{\tilde{b}}(\tilde{c} \overline{\tilde{c}}))}{\Gamma(\tilde{Z} \rightarrow \text { hadrons })} \simeq \frac{\left(g_{L}^{(\tilde{b}, \tilde{c})}\right)^{2}+\left(g_{R}^{(\tilde{b}, \tilde{c})}\right)^{2}}{\sum_{q}\left(g_{L}^{q}\right)^{2}+\left(g_{R}^{q}\right)^{2}} \\
A_{F B}^{b, c} & =\frac{3}{4} \mathcal{A}_{e} \mathcal{A}_{b, c} \simeq \frac{3}{4} \mathcal{A}_{e} \frac{\left(g_{L}^{(\tilde{b}, \tilde{c})}\right)^{2}-\left(g_{R}^{(\tilde{b}, \tilde{c})}\right)^{2}}{\left(g_{L}^{(\tilde{b}, \tilde{c})}\right)^{2}+\left(g_{R}^{(\tilde{b}, \tilde{c})}\right)^{2}} \\
R_{\ell} & \equiv \frac{\Gamma_{\text {hadron }}}{\Gamma_{\ell \ell}}
\end{aligned}
$$

where we have neglected the masses of SM quarks and leptons. We defined the coupling ratio factor:

$$
\mathcal{A}_{f} \equiv \frac{\left(g_{L}^{f}\right)^{2}-\left(g_{R}^{f}\right)^{2}}{\left(g_{L}^{f}\right)^{2}+\left(g_{R}^{f}\right)^{2}}
$$

for any of the SM quarks and leptons. The $\tilde{Z}$ coupling expressions in Eq. (42) has been used. In particular, the coupling between $\tilde{Z}$ and $\tilde{b}$ is changed due to the mixing between $\tilde{K}$ and $\tilde{Z}$

$$
\delta g_{\tilde{Z} \tilde{b}_{R} \tilde{\bar{b}}_{R}} \sim-g_{D} \sin \alpha c_{b(c), R}^{2}
$$

Note that the values of the mixing angles for the bottom and charm quarks with the heavy vectorlike quark are constrained by the requirement of correctly reproducing the bottom and charm mass:

$$
\left|s_{c, L} s_{c, R}\right| \sim \frac{m_{c}}{m_{c, \psi}} \lesssim 5 \times 10^{-4}, \quad\left|s_{b, L} s_{b, R}\right| \sim \frac{m_{b}}{m_{b, \psi}}<2.7 \times 10^{-3},
$$

where we have required the masses of heavy vectorlike quarks to be larger than $1 \mathrm{TeV}$ to satisfy the LHC direct search bounds, and the running mass of the bottom and charm quark at the $1 \mathrm{TeV}$ scale has been used. This makes all mixing angles naturally small and hence the $c_{b,(c), R} \simeq 1$.

In Fig. 1, we present the $1 \sigma$ bounds on the different precision measurements, considering the measurement of $A_{\mathrm{FB}}^{b, c}, R_{b, c, l}$, and $\Gamma_{\text {tot }}$. The constraints coming from different measurements are represented by different colors, and the shaded areas are excluded at the $1 \sigma$ level, with colors corresponding to a superposition of the colors associated to the observables that lead to a constraint in that region of parameters. Most importantly, the white bands are allowed by all precision measurements at the $1 \sigma$ level and can fit the deviation of the forward-backward asymmetry $A_{\mathrm{FB}}^{b}$ within $1 \sigma$. Combing all the electroweak precision measurements and $\mathrm{T}$ parameter constraint, we find out the preferred parameter space of $g_{D}$ and $\sin \alpha$ is

$$
g_{D} \sin \alpha \sim-0.011 .
$$

And we also fix the other mixing angles

$$
\begin{array}{ll}
s_{b, L}=-0.07, & s_{b, R}=-0.001, \\
s_{c, L}=-0.1, & s_{c, R}=-0.001 .
\end{array}
$$

Note that the observables $s_{c(b), R}$ have much weaker impact on the electroweak precision measurement compared with $s_{c(b), L}$, because the $\tilde{Z}$ coupling to SM fermion in Eq. (42) contains $s_{c(b), R}$ only from $\tilde{Z}$ and $\tilde{K}$ mixing. The other change in the coupling come from the left-handed mixing angles $c_{c(b), L}$.

Considering a benchmark point for which $m_{\tilde{K}}=$ $115 \mathrm{GeV}$, the constraint from the measurement of the $T$ parameter requires $|\sin \alpha|<0.064$. Recall the modification of the $Z b_{R} \bar{b}_{R}$ coupling in Eq. (42) neglecting the tiny bottom mixing angles:

$$
\delta g_{\tilde{Z} \tilde{b}_{R} \bar{b}_{R}} \sim-g_{D} \sin \alpha
$$

The $\mathrm{T}$ parameter constraint can also been rewritten as: 

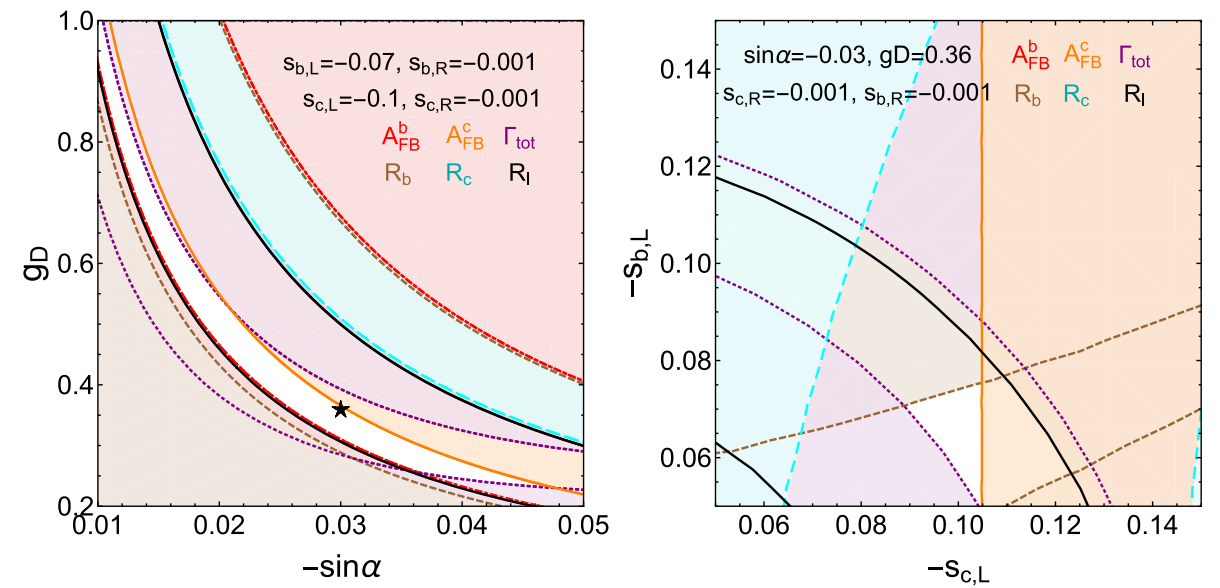

FIG. 1. The color lines represent the $1 \sigma$ bounds on different $\mathrm{Z}$ pole precision observables $R_{b}, R_{c}, R_{L}, A_{F B}^{b}, A_{F B}^{c}$ and $\Gamma_{\text {tot }}$. The color shaded regions are excluded by these measurements at the $1 \sigma$ level. The white areas are the allowed regions by those precision measurements at $1 \sigma$. The black star in the left panel is our benchmark point.

$$
\frac{\left(\delta g_{\tilde{Z} \tilde{b}_{B} \tilde{\tilde{B}}_{R}}\right)^{2}}{g_{D}^{2}} \frac{m_{K}^{2}-m_{Z}^{2}}{m_{Z}^{2}} \in[-0.00121,0.00246]
$$

This clearly put a bound in the $m_{\tilde{K}}-g_{D}$ plane for fixed value of $\delta g_{\tilde{Z} \tilde{b}_{R} \tilde{\tilde{b}}_{R}}$, which is shown as orange region in Fig. 3 for $\delta g_{\tilde{Z} \tilde{b}_{R} \tilde{b}_{R}}=0.011$. Note such value can solve the $A_{F B}^{b}$ discrepancy. We can see clearly that the constraints on the T parameter almost exclude the lower half of the parameter space. Since we will take $g_{D}, m_{\tilde{K}}, \sin \alpha$ as input parameters, the $c_{\beta}^{2}$ can be written as:

$$
c_{\beta}^{2} \sim-\frac{\Delta m_{Z}^{2}}{m_{Z}^{2}} \frac{\sqrt{g^{2}+g^{\prime 2}}}{2 \delta g_{\tilde{Z} \tilde{b}_{R} \tilde{b}_{R}}},
$$

where we can easily see that in order to modify $A_{\mathrm{FB}}^{b}$ at the desired value and be consistent with $\mathrm{T}$ parameter constraint, we need $c_{\beta}^{2} \lesssim 0.08$. It indicates the vev of $\Phi_{1}$ should be small, i.e. $v_{1} \lesssim 75 \mathrm{GeV}$.

\section{IV. $\tilde{K}$ SEARCHES AT COLLIDERS}

In this section, we will consider the phenomenology of $\tilde{K}$ at the LHC. Since our $\tilde{K}$ only coupled with bottom and charm quarks before the small mixing between the $\tilde{Z}$ boson, its main production channel will be $\tilde{b} \overline{\tilde{b}}$ and $\tilde{c} \overline{\tilde{c}}$ initiated processes. It will also mainly decay into bottom and charm quarks with roughly the same branching ratio $\sim 50 \%$. The decay into leptons will be highly suppressed by the small mixing. We present the decay branching ratios of $\tilde{K}$ in Fig. 2. There could be another decay channel of $\tilde{K} \rightarrow \chi_{2} \bar{\chi}_{2}$ if $m_{\chi_{2}}<m_{\tilde{K}} / 2$, which would be around $1 / 7$ due to the color factor counting in low mass limit.

The presence of the light gauge boson $\tilde{K}$ is subject to several constraints. The first constraint comes from the

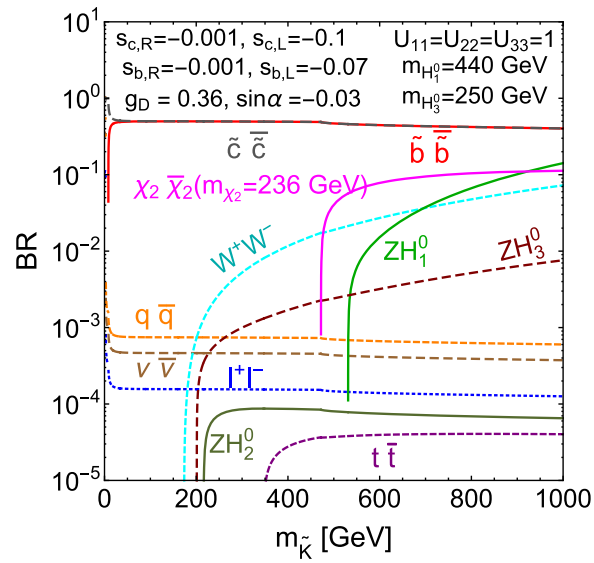

FIG. 2. The decay branching ratio for $\tilde{K}$. Left panel is for low mass $\chi_{2}$ and right panel is for high mass $\chi_{2}$, which are two DM benchmarks in Sec. V. 
exotic $Z^{\prime}$ decaying to dijet which associated produced with a jet from CMS [26] at $13 \mathrm{TeV}$, which is shown as red region in Fig. 3. We see that there is a deep valley around $115 \mathrm{GeV}$, which is associated with an interesting $2.9 \sigma$ local excess in that region of invariant masses. CMS and ATLAS also search for exotic $Z^{\prime}$ decay to b-jet pair [27,28] at $13 \mathrm{TeV}$, but focus on the mass region around 550-1500 GeV. We only show the constraint from CMS as the blue region in Fig. 3 since CMS present the constraint on the cross-section directly. The parameter spaces considered by ATLAS and CMS are not relevant to our analysis since they were already excluded by the $T$ parameter constraints, when the $A_{F B}^{b}$ anomaly is considered by requiring $g_{D} \sin \alpha=-0.011$.

The next constraint is the exotic $Z^{\prime}$ leptonic decay. We consider all the corresponding LHC searches at $7 \mathrm{TeV}$ [31], $8 \mathrm{TeV}$ [32], $13 \mathrm{TeV}$ [30,33], and also the Tevatron $1.96 \mathrm{TeV}$ searches by D0 and CDF [34,35]. Comparing all the searches, the most stringent constraint comes from the $13 \mathrm{TeV}$ ATLAS search [30] (green shaded) which goes down to $170 \mathrm{GeV}$. The constraints from D0 and CDF are shown as brown and cyan area. We also show $\mathrm{T}$ parameter constraint in Fig. 3 as orange area.

LHC also searched for the low mass scalar in its leptonic decay. For our benchmark point, the branching ratio of $\tilde{K}$ to

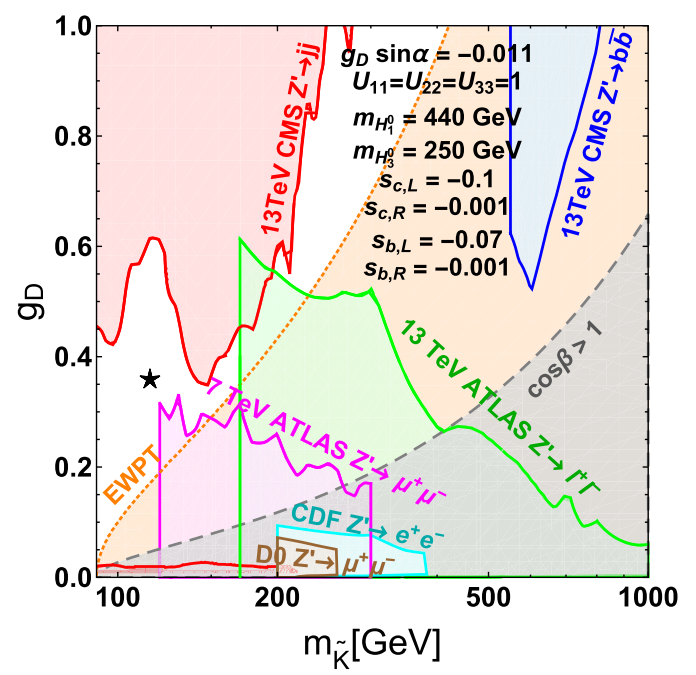

FIG. 3. The constraints from collider searches on $\tilde{K}$ for $g_{D} \sin \alpha=-0.011$. The mixing angle between heavy vectorlike quarks and SM $b, c$ quarks are chosen following Fig. 1, where the EWPT constraint is the T parameter constraint under such choice. The red (blue) shaded regions correspond to exotic $Z^{\prime}$ search in dijet (b-jet pair) channel from CMS at $13 \mathrm{TeV}$ [26] ([28]), labeled as "13 TeV CMS $Z^{\prime} \rightarrow j j$ ” ("13 TeV CMS $Z^{\prime} \rightarrow \bar{b} b$ "). The $Z^{\prime} \rightarrow$ $\ell^{+} \ell^{-}$constraints from $\mathrm{D} 0$ and $\mathrm{CDF}$ are shown as brown and cyan area, labeled as "D0 $Z^{\prime} \rightarrow \mu^{+} \mu^{-}$" and "CDF $Z^{\prime} \rightarrow e^{+} e^{-}$". The $Z^{\prime}$ dilepton searches at LHC are shown as magenta and green shaded area, from $7 \mathrm{TeV}$ [29] and $13 \mathrm{TeV}$ [30] ATLAS, labeled as "7 TeV ATLAS $Z^{\prime} \rightarrow \mu^{+} \mu^{-}$" and " $13 \mathrm{TeV}$ ATLAS $Z^{\prime} \rightarrow \ell^{+} \ell^{-}$". The gray region is excluded because $\cos \beta>1$, while above the line has $\cos \beta<1$. $e^{+} e^{-}, \mu^{+} \mu^{-}$, and $\tau^{+} \tau^{-}$are the same, which is $8.7 \times 10^{-5}$. The most recently research is done by ALTAS [29] at $7 \mathrm{TeV}$ and the constraint is $\sim 0.1 \mathrm{pb}$ around mass $m_{\phi}=120 \mathrm{GeV}$, which is the lowest mass they considered in the $\mu^{+} \mu^{-}$ channel. For our benchmark point, the cross section for $p p \rightarrow\left(\tilde{K} \rightarrow \mu^{+} \mu^{-}\right)=0.08 \mathrm{pb}$ at $m_{\tilde{K}}=115 \mathrm{GeV}$, which is again marginal within the constraint from ATLAS. The constraint is shown as the magenta shaded area of Fig. 3.

Before closing this section, we comment on the intriguing hints of lepton flavor non-universality observed in the $R_{K}^{(*)}$ $[36,37]$ processes at the LHCb experiment and also in $R_{D}^{(*)}$ processes at the BABAR experiment [38,39] and at $\mathrm{LHCb}$ [40] in charged lepton decay channel with tau leptons, though only weakly supported by Belle $[41,42]$ and the recent LHCb result [43] from three-prong tau lepton decays.

In our model, the gauge boson $K$ couples flavor diagonally to $b$ and $c$ quark and hence not in a flavor universal way, which is similar to Refs. [44,45]. In this case, the $W^{ \pm}$loop effects can introduce flavor changing coupling between the $K$ boson and $b, s$ quarks. However, the leptons couple with $K$ only via $Z$ boson mixing, and hence the gauge boson couplings are lepton flavor universal. Therefore, our model is unlikely to address $R_{K}^{(*)}$, unless we introduce, for example, muon leptons charged under $U(1)_{D}$. Thus, it needs further considerations to reconcile $R_{K}$ or $R_{K}^{*}$ problems with bottom quark forward-backward asymmetry problem, what is beyond the scope of this paper. For $R_{D}^{(*)}$ lepton flavor nonuniversality, the charged Higgs extension in type-II $2 \mathrm{HDM}$ has been excluded by the combination of $R_{D}$ and $R_{D}^{*}$ [38]. In our model, the $U(1)_{D}$ assignment of $\Phi_{1}$ determines that it is similar to type-I 2HDM. In this case, the charged Higgs coupling to quarks are suppressed by $\cot \beta$, which we take to be small, and its contributions would be further suppressed by the fact that the masses of our Higgs bosons $H_{0}, A_{0}$ and $H^{ \pm}$are large, of order of a few to several $\mathrm{TeV}$, which further reduces their relevance to $R\left(D^{*}\right)$. Actually one might try changing $U(1)_{D}$ charge of $\Phi_{1}$ from $X$ to $-X$ in order to write down SM Yukawa coupling for $\Phi_{1}$. However, this induces the wrong $\operatorname{sign}$ for $\sin \alpha$, which forces us to stay with the current charge assignment in Table I. Therefore, we conclude that an extension of this model would be necessary to solve the flavor problems in $R_{K}^{(*)}$ and $R_{D}^{(*)}$ together with the bottom-quark forward-backward asymmetry. We shall not explore such an extension in this article.

\section{A. Benchmark for bottom-quark forward-backward asymmetry}

Based on the constraints from electroweak precision measurement and $\tilde{K}$ search at LHC, we set our benchmark point as $m_{\tilde{K}}=115 \mathrm{GeV}, g_{D}=0.36$, and $\sin \alpha=-0.03$, resulting $\tan \beta=7.4$. The choice of $g_{D}$ and $\sin \alpha$ satisfies the constraints from Z-pole observables in Fig. 1, which 
TABLE II. The cross sections for $\tilde{K}$ production in Drell-Yan channel and jet associated channels. For the cross section calculation, the mixing between $\tilde{K}$ and $\tilde{Z}$ is not included due to small value of $\sin \alpha$.

\begin{tabular}{lccc}
\hline \hline$g_{D}=0.36, \sin \alpha=-0.03$ & $7 \mathrm{TeV}$ & $8 \mathrm{TeV}$ & $13 \mathrm{TeV}$ \\
\hline$\sigma(p p \rightarrow \tilde{K})[\mathrm{pb}]$ & $1.0 \times 10^{3}$ & $1.3 \times 10^{3}$ & $3.1 \times 10^{3}$ \\
$\sigma(p p \rightarrow \tilde{K} j)[\mathrm{pb}]$ & $3.6 \times 10^{2}$ & $4.8 \times 10^{2}$ & $1.3 \times 10^{3}$ \\
$\sigma(p p \rightarrow \tilde{K} j j)[\mathrm{pb}]$ & $1.3 \times 10^{2}$ & $1.8 \times 10^{2}$ & $6.7 \times 10^{2}$ \\
\hline \hline
\end{tabular}

especially can also lead to $1 \sigma$ agreement for the bottomquark forward-backward asymmetry. In Fig. 3, the collider limits of $\tilde{K}$ and T parameter still allow its mass to be around $[100,140]$. We do not consider degenerate masses between $Z$ and $K$ which may leads to large mixing. $m_{\tilde{K}}=115$ is chosen because of the interesting $2.9 \sigma$ local excess in Ref. [26], but other $m_{\tilde{K}}$ around this region is also plausible.

Note that the mass of the new gauge boson $\tilde{K}$ is very close to SM Higgs mass. For this benchmark point, the Drell-Yan cross section for $\tilde{K}$ production at the $13 \mathrm{TeV}$ LHC will be sizable, around $3.1 \times 10^{3} \mathrm{pb}$. The associated production cross section at LHC with another one or two jets are also listed in Table II.

For our benchmark point $m_{\tilde{K}}=115 \mathrm{GeV}$, since it can decay into $\tilde{b} \overline{\tilde{b}}$ at around $50 \%$, it can easily fake a $b \bar{b}$ decaying SM Higgs boson $m_{h}=125 \mathrm{GeV}$ at the LHC, because the large uncertainty for reconstructing hadronically decaying particles. In this case, it is important to check the constraints coming from SM Higgs searches with the Higgs decaying into bottom quark pairs. To calculate the cross-sections in our model, we have used FeYnRules 2.0 [46] to generate the model files and implement it in MADGRAPH5_aMC@NLO [47]. The cross-sections are calculated by MADGRAPH5 at treelevel to estimate the constraints.

We first consider the SM Higgs searches from VBF production by ATLAS at the $8 \mathrm{TeV}$ [48] and $13 \mathrm{TeV}$ [49] and also by CMS at the $8 \mathrm{TeV}$ [50]. The observed 95\% upper limit on SM Higgs cross section times the branching ratio is $4.1 \mathrm{pb}$ from ATLAS and $4.6 \mathrm{pb}$ from CMS at $8 \mathrm{TeV}$. For our benchmark point, the cross section for the process $p p \rightarrow j j \tilde{K}=162 \mathrm{pb}$ with $p_{T, j}>20 \mathrm{GeV},\left|\eta_{j}\right|<5$. In order to obtain the rough idea about the constraint by comparing the LCH VBF search, we simplify require $m_{j j}>650 \mathrm{GeV}$ from the Madgraph parton-level simulation for the SM VBF Higgs process and for our $\tilde{K} j j$. This cut efficiency for $\tilde{K} j j$ is only 0.006 comparing to the cut efficiency on SM VBF process 0.23. Then the effective cross section after this cut for our benchmark point is only $\sigma(p p \rightarrow j j(\tilde{K} \rightarrow b \bar{b})) \sim 0.5$ pb by including the branching ratio of $\tilde{K} \rightarrow b \bar{b}$, which is smaller than the constraint from ATLAS [48] 0.94 and $1.06 \mathrm{pb}$ from CMS [50].

At $13 \mathrm{TeV}$, ATLAS collaboration has explored SM Higgs in VBF production with an associated high energy photon in [49]. The observed 95\% C.L. upper limit on the production cross section times branching ratio for a Higgs mass of $125 \mathrm{GeV}$ is 4.0 times the Standard Model expectation. We use MADGRAPH to produce SM $H j j \gamma$ and our model $\tilde{K} j j \gamma$, with both $H$ and $\tilde{K}$ decaying to $\bar{b} b$. At the parton level, we estimate the cross section based on the basic cuts $p_{T}^{j}>40 \mathrm{GeV}, p_{T}^{\gamma}>30 \mathrm{GeV}$, and $m_{j j}>800 \mathrm{GeV}$. After cuts and multiplying the corresponding $\bar{b} b \mathrm{BR}$, we found $\mathrm{SM} \mathrm{Hjj \gamma} \rightarrow \bar{b} b j j \gamma$ and $\tilde{K} j j \gamma \rightarrow \bar{b} b j j \gamma$ have cross sections of about 4.5 and $4.0 \mathrm{fb}$ respectively. Therefore, we conclude that our benchmark is not excluded by the constraints coming from the SM Higgs search in the VBF channel with an associated high energy photon.

Besides the VBF search, LHC also searched for SM Higgs via $Z H$ and $W^{ \pm} H$ associated production. The constrain on such scenario is $\sigma(Z H) \times \mathrm{BR}(H \rightarrow b \bar{b})=$ $0.57_{-0.23}^{+0.26} \mathrm{pb}$ from ATLAS [51]. For our model, the corresponding process is $p p \rightarrow \tilde{Z} \tilde{K}$, the cross section is suppressed by $\sin ^{2} \alpha \sim 10^{-3}$, which is much smaller than the SM cross section.

Before closing the section, we make some more comments on the $2.9 \sigma$ excess in the di-jet resonance searches at $13 \mathrm{TeV}$ CMS [26], which motivated us to set $m_{\tilde{K}}=$ $115 \mathrm{GeV}$ as the benchmark point. This search is dedicated to look for new vector resonance $Z^{\prime}$, which only coupled to the SM quarks with universal vectorlike coupling, and the largest deviation from the SM background only hypothesis is around $m_{Z^{\prime}}=115 \mathrm{GeV}$ with local significance $\sim 2.9 \sigma$. Comparing the observed $95 \%$ C.L. upper limit cross section $\sim 1.05 \times 10^{4} \mathrm{pb}$ for the $Z^{\prime}$ with the expected one $\sim 4.5 \times 10^{3} \mathrm{pb}$, we can see that roughly one needs $5 \times 10^{3} \mathrm{pb}$ to fit the excess. The cross section in our benchmark point at tree level is $3.1 \times 10^{3} \mathrm{pb}$, which is capable to explain this excess. The search requires high $p_{T}$ $Z^{\prime}$ that the dijet merged into a single jet. Given that in our model, $\tilde{K}$ decays to $\bar{b} b$ and $\tilde{c} c$ at equal rate, it is interesting to analyze what could be the significance had CMS performed heavy flavor tagging, something not done in Ref. [26]. At $13 \mathrm{TeV}$ LHC [52], CMS collaboration has looked for the high $p_{T}$ fat jet with b-tagging in the inclusive $H+j$ measurement. The tagging efficiency is $33 \%$ for $H \rightarrow(b \bar{b})$ as a fat jet and $1 \%$ for mistagging efficiency from light flavor quarks. If applying b-tagging in $Z^{\prime}$ resonance search in Ref. [26], the increase in $S / \sqrt{B}$ is $50 \% \times 33 \% / \sqrt{1 \%} \sim 1.6$ which is a moderate increase if the background error is statistic dominant.

The CMS collaboration further used this high $p_{T}$ fat jet with b-tagging technique in related searches for the inclusive $H+j$ process with $H \rightarrow \bar{b} b$, by requiring $p_{T}^{H}>$ $450 \mathrm{GeV}$ [52]. The theoretical cross section for $H(\bar{b} b)$ with $p_{T}^{j}>450 \mathrm{GeV}$ is $31.7 \pm 9.5 \mathrm{fb}$ with $30 \%$ uncertainty, while the measured value is $74 \pm 50 \mathrm{fb}$. The mean value 
is therefore about 2.5 times the Higgs one, with an observed significance of $1.5 \sigma$. No other significant resonances have been found. In our benchmark model, the cross sections after cut for $\tilde{K} j \rightarrow(b b) j$ and $\tilde{K} b \rightarrow(b b) b$ are about 41 and $25 \mathrm{fb}$, respectively. Note that $K j$ has a similar cross-section as $H j$, and $m_{\tilde{K}}=115 \mathrm{GeV}$ in our benchmark. Moreover, with an extra b quark in $\tilde{K} b$, the misreconstruct, miscombination, and mistagging might result in a smaller contribution, thus we estimate its contribution should be less significant. The $m_{b b}$ distribution in Fig. 4 of [52] presents a broad excess that range from 105 to $140 \mathrm{GeV}$ and therefore, although a dedicated experimental analysis must be performed, we conclude that the $\tilde{K}$ signal is compatible with the current experimental observations in this channel. Higher luminosity LHC measurements in this channel are likely to provide the most effective way of probing this scenario.

\section{DARK MATTER SEARCH}

In this section, we will explore in detail the possibility of the neutral vectorlike fermion $\chi_{2}$ being a dark matter candidate. The interaction Lagrangian for $\chi_{2}$ in the mass basis at leading order of $\sin \alpha$ and $\cos \beta$ is

$$
\begin{aligned}
\mathcal{L}_{\chi_{2}} \simeq & -g_{D} \cos \alpha \tilde{K}_{\mu} \bar{\chi}_{2, R} \gamma^{\mu} \chi_{2, R}+g_{D} \sin \alpha \tilde{Z}_{\mu} \bar{\chi}_{2, R} \gamma^{\mu} \chi_{2, R} \\
& +\frac{m_{\chi_{2}}}{v_{D}} \bar{\chi}_{2} \chi_{2}\left(\frac{v}{v_{D}} \cos \beta H_{1}^{0}+U_{23} H_{2}^{0}-U_{33} H_{3}^{0}\right) \\
& +i \frac{m_{\chi_{2}} v}{v_{D}^{2}} \cos \beta A^{0} \bar{\chi}_{2} \gamma_{5} \chi_{2},
\end{aligned}
$$

where $m_{\chi_{2}}=y_{\chi_{2}} v_{D} / \sqrt{2}$. The Majorana mass term $\frac{1}{2} M_{m} \bar{\chi}_{2, L} \chi_{2, L}^{c}$ in Eq. (10) will split the Dirac fermion into two Majorana fermions, which is similar to the inelastic DM setup considered in Ref. [53]. In the Weyl fermion basis $\left(\chi_{2, L}, \chi_{2, R}^{c}\right)^{T}$, the mass matrix is given by:

$$
M_{\chi}=\left(\begin{array}{cc}
M_{m} & m_{\chi_{2}} \\
m_{\chi_{2}} & 0
\end{array}\right),
$$

where we assume $M_{m}, m_{\chi_{2}}>0$ without loss of generality. This symmetric mass matrix can be diagonalized by an orthogonal rotation:

$$
\left(\begin{array}{c}
\chi_{2, L} \\
\chi_{2, R}^{c}
\end{array}\right)=U_{\chi_{2}}\left(\begin{array}{c}
\eta_{1} \\
-i \eta_{2}
\end{array}\right)=\left(\begin{array}{cc}
c_{\chi_{2}} & s_{\chi_{2}} \\
-s_{\chi_{2}} & c_{\chi_{2}}
\end{array}\right)\left(\begin{array}{c}
\eta_{1} \\
-i \eta_{2}
\end{array}\right)
$$

where $\eta_{1,2}$ are the mass eigenstates of two Majorana fermions and the factor $-i$ is to ensure the Majorana masses of $\eta_{1,2}$ have the same value $m_{\chi_{2}}$ in the limit of $M_{m}=0$.

In the small Majorana mass limit $M_{m} \ll m_{\chi_{2}}$, the eigenstate masses are

$$
\begin{aligned}
& m_{\eta_{1}}=m_{\chi_{2}}+\frac{M_{m}}{2}, \\
& m_{\eta_{2}}=m_{\chi_{2}}-\frac{M_{m}}{2},
\end{aligned}
$$

where the mixing angle is given by:

$$
\begin{gathered}
c_{\chi_{2}}=\frac{1}{\sqrt{2}}+\frac{M_{m}}{4 \sqrt{2} m_{\chi_{2}}} \simeq \frac{1}{\sqrt{2}}, \\
s_{\chi_{2}}=-\frac{1}{\sqrt{2}}+\frac{M_{m}}{4 \sqrt{2} m_{\chi_{2}}} \simeq-\frac{1}{\sqrt{2}} .
\end{gathered}
$$

For large Majorana mass $M_{m} \gg m_{\chi_{2}}$, the eigenstate masses are

$$
\begin{gathered}
m_{\eta_{1}}=M_{m}+\frac{m_{\chi_{2}}^{2}}{M_{m}} \\
m_{\eta_{2}}=\frac{m_{\chi_{2}}^{2}}{M_{m}}
\end{gathered}
$$

which is a typical see-saw mass, with the mixing angle $s_{\chi_{2}}=-m_{\chi_{2}} /\left(M_{m}\right) \ll 1$. With the mixing angle we can rewrite the light Majorana DM $\eta_{2}$ back into its Dirac form,

$$
\chi_{2}^{\prime}=\left(\begin{array}{c}
i \eta_{2} \\
-i \eta_{2}^{\dagger}
\end{array}\right)
$$

and also the interaction Lagrangian as follows:

$$
\begin{aligned}
\mathcal{L}_{\chi_{2}^{\prime}} \simeq & -g_{D} \cos \alpha c_{\chi_{2}}^{2} \tilde{K}_{\mu} \bar{\chi}^{\prime}{ }_{2} \gamma^{\mu} \gamma_{5} \chi_{2}^{\prime}+g_{D} \sin \alpha c_{\chi_{2}}^{2} \tilde{Z}_{\mu} \bar{\chi}^{\prime}{ }_{2} \gamma^{\mu} \gamma_{5} \chi_{2}^{\prime} \\
& +\frac{m_{\chi_{2}}}{v_{D}} 2 s_{\chi_{2}} c_{\chi_{2}} \bar{\chi}^{\prime}{ }_{2} \chi_{2}^{\prime}\left(\frac{v}{v_{D}} \cos \beta H_{1}^{0}+U_{23} H_{2}^{0}-U_{33} H_{3}^{0}\right) \\
& +i 2 s_{\chi_{2}} c_{\chi_{2}} \frac{m_{\chi_{2}} v}{v_{D}^{2}} \cos \beta A^{0} \bar{\chi}^{\prime}{ }_{2} \gamma_{5} \chi_{2}^{\prime} .
\end{aligned}
$$

We can simplify it by

$$
\mathcal{L}_{\chi_{2}^{\prime}} \simeq\left\{\begin{array}{ll}
\frac{-g_{D}}{2} \bar{\chi}^{\prime}{ }_{2} \gamma^{\mu} \gamma_{5} \chi_{2}^{\prime}\left(\cos \alpha \tilde{K}_{\mu}+\sin \alpha \tilde{Z}_{\mu}\right)-\frac{m_{\chi_{2}}}{v_{D}} \bar{\chi}_{2}{ }_{2} \chi_{2}^{\prime} U_{33} H_{3}^{0} & \left(M_{m} \ll m_{\chi_{2}}\right) \\
-g_{D} \bar{\chi}^{\prime}{ }_{2} \gamma^{\mu} \gamma_{5} \chi_{2}^{\prime}\left(\cos \alpha \tilde{K}_{\mu}+\sin \alpha \tilde{Z}_{\mu}\right) & \left(M_{m} \gg m_{\chi_{2}}\right)
\end{array},\right.
$$


where we keep only the leading order interactions in $\mathcal{O}\left(M_{m} / m_{\chi_{2}}\right)$ or $\mathcal{O}\left(m_{\chi_{2}} / M_{m}\right)$. In this following subsections, we will discuss the phenomenology of Dirac and Majorana DM separately.

\section{A. Dirac dark matter}

We first consider the case of pure Dirac dark matter, whose Lagrangian is listed in Eq. (65). We will study the condition to obtain the correct relic abundance and explore the dark matter limits from indirect detection, direct detection and collider searches.

\section{DM annihilation}

We first calculate the $\chi_{2} \chi_{2}$ annihilation cross sections. The DM annihilation $\bar{\chi}_{2} \chi_{2} \rightarrow \bar{f} f$ is an s-channel process, mediated by $\tilde{K}, \tilde{Z}, H_{1,2,3}^{0}$ and $A^{0}$. From Eq. (65), only processes with $b \bar{b}(c \bar{c})$ final states and mediated by $\tilde{K}$ and $H_{3}^{0}$ are not suppressed by small mixing angle $\sin \alpha$ and $\cos \beta$. Given that the Yukawa couplings between $H_{3}^{0}$ and $b$, $c$ quarks are much smaller than 1 , we conclude that the dominant DM annihilation process is $\overline{\chi_{2}} \chi_{2} \rightarrow \tilde{K}^{*} \rightarrow \bar{b} b, \bar{c} c$ with annihilation cross section

$$
\begin{aligned}
(\sigma v)_{\chi_{2} \bar{\chi}_{2} \rightarrow \bar{q} q}^{q=b, c} & \\
= & \frac{g_{D}^{4} \sqrt{1-\frac{4 m_{q}^{2}}{s}}}{8 \pi\left(\left(s-m_{\tilde{K}}^{2}\right)^{2}+m_{\tilde{K}}^{2} \Gamma_{\tilde{K}}^{2}\right)} \\
& \times\left[s-m_{q}^{2}+m_{\chi_{2}}^{2}\left(-1+\frac{m_{q}^{2}\left(4 m_{\tilde{K}}^{4}-6 m_{\tilde{K}}^{2} s+3 s^{2}\right)}{m_{\tilde{K}}^{4} s}\right)\right] \\
\approx & \frac{g_{D}^{4}}{8 \pi\left(\left(s-m_{\tilde{K}}^{2}\right)^{2}+m_{\tilde{K}}^{2} \Gamma_{\tilde{K}}^{2}\right)}\left(s-m_{\chi_{2}}^{2}\right)
\end{aligned}
$$

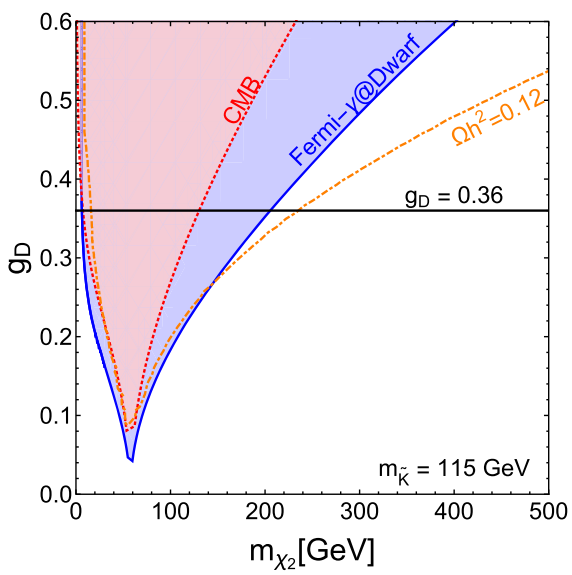

where we have neglected the quark mass in the second line. For the annihilation at freeze-out, it needs to be averaged over thermal distribution of DM, while for annihilation today, it only needs the substitution $s=4 m_{\chi_{2}}^{2}$.

To reproduce the right relic abundance $\Omega h^{2}=0.12$ [54], the thermal averaged cross-section for Dirac fermion DM is about $6 \times 10^{-26} \mathrm{~cm}^{3} / \mathrm{s}$. In Fig. 4, we plot the contours (the orange line) in the $m_{\chi_{2}}-g_{D}$ plane, which gives the right relic abundance for our benchmark scenario $m_{\tilde{K}}=115 \mathrm{GeV}$. If we further choose $g_{D}=0.36$ as our benchmark point, we obtain two solutions for the DM mass, $m_{\mathrm{DM}}=14 \mathrm{GeV}$ or $236 \mathrm{GeV}$, which can satisfy the relic abundance requirement.

\section{DM indirect detection}

The Dirac fermion DM $\chi_{2}$ annihilation to $\bar{b} b$ and $\bar{c} c$ have equal rate, with total annihilation cross-section leading to right relic abundance for DM mass 14(236) GeV. Since the annihilation is s-wave, the final state particles from DM annihilation will inject energy into primordial plasma which would delay recombination and thus leave observable imprints in the cosmic microwave background (CMB) [59-62]. Given that energy injection efficiency of $\bar{b} b$ and $\bar{c} c$ are similar [63], the constraint from CMB [54] is

$p_{\text {ann }}(z) \equiv f(z) \frac{\langle\sigma v\rangle}{m_{\chi_{2}}}<3.5 \times 10^{-28} \mathrm{~cm}^{3} \mathrm{~s}^{-1} \mathrm{GeV}^{-1}$,

Making use of the $f(z)$ function from [62], we plot the excluded region (in red) in Fig. 4, where we can see that the low mass benchmark $m_{\chi_{2}}=14 \mathrm{GeV}$ is excluded, while the high mass $m_{\chi_{2}}=236 \mathrm{GeV}$ is still allowed.

In addition, the Fermi-LAT gamma-ray observations of dwarf galaxies provide a constraint on the DM annihilation

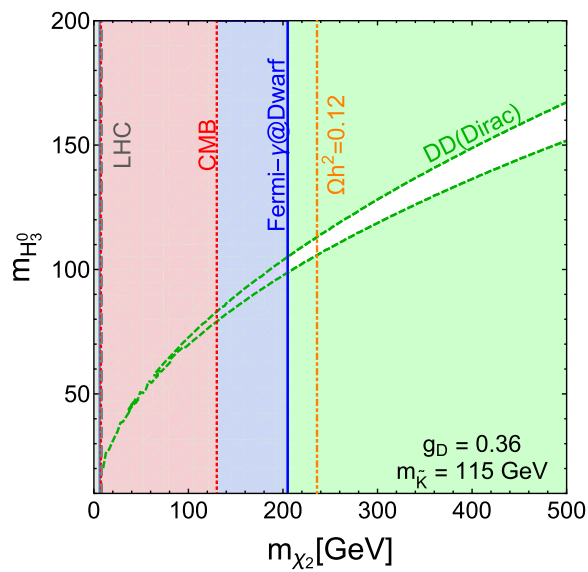

FIG. 4. The constraints on Dirac dark matter parameter space in $m_{\chi_{2}}-g_{D}$ plane (Left) and $m_{\chi_{2}}-m_{H_{3}^{0}}$ plane (Right). The orange line correspond to parameters that lead to the right relic abundance $\Omega h^{2}=0.12$. The red shaded region gives the CMB limits [54], while the blue shaded region gives the most stringent gamma-ray limits from Fermi observation in dwarf galaxies $[55,56](1 a b e l e d$ as "Fermi $\gamma$ at Dwarf galaxies"). The green area is excluded by Xenon1T [57] for benchmark point parameters. The gray line is limits from jets + MET with $1 \mathrm{~b}$-jet tagging at $13 \mathrm{TeV}$ CMS [58]. 
cross sections based on final states [55,56]. For $\bar{b} b$ final states, this tells us that the DM mass should be larger than $100 \mathrm{GeV}$, i.e. $m_{\chi_{2}} \gtrsim 100 \mathrm{GeV}$, in order to have the right thermal relic density. Since the photon spectrum from final state $\bar{b} b$ and $\bar{c} c$ are quite similar [64], it again excludes the light DM benchmark but not for the heavy one. The gamma-ray observation from Galactic center (GC) by Fermi-LAT gives constraint $m_{\mathrm{DM}} \gtrsim 50 \mathrm{GeV}$ for $\bar{b} b$ final states [65], which is less stringent than dwarf galaxies. There is also a gamma-ray constraint from the Virgo cluster [66], but is much weaker than the above two constraints. Therefore, in Fig. 4, we only show the most stringent limits from Fermi dwarf galaxies observation in blue shaded area.

\section{DM direct detection}

In this section, we will consider the direct detection (DD) of $\chi_{2}$, which are related to the scattering between $\chi_{2}$ and nucleon. The sum of different flavor quark contribution inside nucleon from scalar mediator should be performed at the amplitude level and the results read:

$$
a_{N}=\left(\sum_{q=u, d, s} f_{\mathrm{Tq}}^{(N)} \frac{a_{q}}{m_{q}}+\frac{2}{27} f_{\mathrm{TG}}^{(N)} \sum_{q=c, b, t} \frac{a_{q}}{m_{q}}\right) m_{N},
$$

where $f_{\mathrm{TG}}^{(N)}, f_{T q}^{(N)}$ are the form factors and $N=p, n$ is proton and neutron respectively. The quark form factors for proton are $f_{\mathrm{Tu}}^{(p)}=0.017 \pm 0.008, f_{\mathrm{Td}}^{(p)}=0.028 \pm 0.014$, $f_{\mathrm{Ts}}^{(p)}=0.040 \pm 0.020, f_{\mathrm{TG}}^{(p)} \approx 0.91[67,68]$ and for neutron are $f_{\mathrm{Tu}}^{(n)}=0.011 f_{\mathrm{Td}}^{(n)}=0.0273, f_{\mathrm{Ts}}^{(n)}=0.0447, f_{\mathrm{TG}}^{(n)} \approx$ 0.917 [69] (see also results from [70,71]). In our model, the scattering between nucleon and $\chi_{2}$ are mediated by $C P$ even scalars $H_{1,2,3}^{0}, C P$-odd scalar $A^{0}$, and neutral gauge boson $\tilde{K}$ and $\tilde{Z}$. We will consider the scalar and vector contribution separately in the next two paragraphs.

For scalar contribution shown in panel (a) of Fig. 5, the scattering between $\chi_{2}$ and light flavor quark mediated via $H_{2}^{0}, H_{3}^{0}$ is suppressed by $\cot \beta$, where the suppression for $H_{2}^{0}$ is from $\chi_{2}$ Yukawa vertex and for $H_{3}^{0}$ is from light flavor $q$ Yukawa vertex, both from $U_{23}$ mixing. The $\chi_{2}$ and light quark scattering mediated by $H_{1}^{0}, A^{0}$ are suppressed by $\cot ^{2} \beta$. Therefore, only the scattering process with heavy flavor quark $b, c$ mediated by $H_{3}^{0}$, are not suppressed by scalar mixing $\sim \cot \beta$ and quark mixing angles. The leading contribution to spin-independent (SI) search is thus $H_{3}^{0}$ mediation via quark form factor $f_{\mathrm{TG}}^{N}$, with the amplitude $a_{q}$ proportional to $m_{\tilde{b}(c)}$. Given that $f_{\mathrm{TG}}^{(p)} \approx f_{\mathrm{TG}}^{(n)}$, the scalar contribution of $H_{3}^{0}$ are isospin universal.

For vector mediation by $\tilde{K}$ and $\tilde{Z}$, we only consider the vector-vector $(\mathrm{V}-\mathrm{V})$ fermion bilinear coupling contribution for SI interaction, shown in panel (b) and (c) in Fig. 5. The reason is that vector-axial (V-A) and axial-vector (A-V) fermion bilinear are further suppressed by powers of velocity or momentum transfer, while axial-axial (A-A) interaction contributes to spin-dependent interaction which is less constraining than SI interaction [72,73]. There are two kinds of contribution to the $\chi_{2}$ and nucleon $\mathrm{V}-\mathrm{V}$ scattering. The first one is DM current couples to $J_{Z}$ current in $\mathrm{SM}, J_{\chi_{2}, \mu} J_{Z}^{\mu}$, which is suppressed by $\tilde{K}, \tilde{Z}$ mixing, shown as panel (b) in Fig. 5. The second one is DM current interaction with $b, c$ quark currents mediated by kinetic mixing $\tilde{K}_{\mu \nu} B^{\mu \nu}$ where $B$ is hypercharge field, which is mixing suppression free, shown as panel (c) in Fig. 5. Similarly like gluon form factor in nucleon for scalar interaction, the second one can induce a coupling to light quark in the nucleon via electromagnetic current $\epsilon e J_{\mathrm{EM}}$, by 1-loop contribution from $b, c$ quark. The kinetic mixing parameter $\epsilon \approx g_{D} g^{\prime} /\left(16 \pi^{2}\right) \sim 10^{-3}$, while given $\sin \alpha \sim 0.03$ in our benchmark, we have $\sin \alpha g / c_{w} \gg \epsilon e$. Thus, it is reasonable to ignore the contribution shown in Fig. 5(c).

In the nonrelativistic and heavy DM limit, both scalar mediation and $\mathrm{V}-\mathrm{V}$ mediation have the fermion bilinear $\overline{\chi_{2}}\left(1+\gamma^{0}\right) \chi_{2} \bar{N} N / 2$, and we can calculate the SI crosssection for $\chi_{2}$ scattering with nucleon $N$ [72]. Note that the $J_{Z}$ current involves an isospin violating coupling that $f_{p}=\frac{g}{4 c_{w}}\left(1-4 s_{w}^{2}\right)$ and $f_{n}=\frac{g}{4 c_{w}}(-1)$, therefore we should average over proton and nucleon in the nuclei. The averaged SI cross-section for $\chi_{2}$ and nucleon is

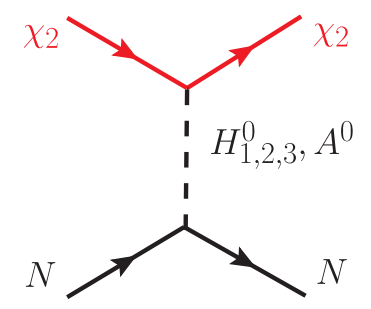

(a)

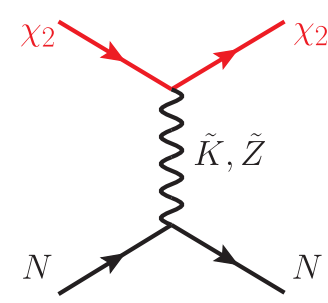

(b)

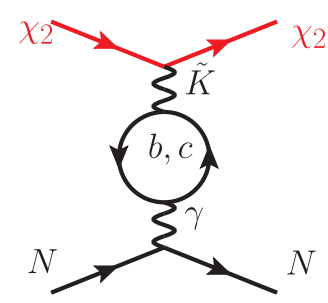

(c)

FIG. 5. The Feynman diagrams for Dirac $\chi_{2}$ scattering with nucleon. The processes in panel (a) and (b) are mediated by scalars and vectors, while (c) is by kinetic mixing of gauge boson at 1-loop. The dominant contributions come from $H_{3}^{0}$ in (a) and $\tilde{K}, \tilde{Z}$ in (b). 


$$
\begin{aligned}
\sigma_{N}^{\mathrm{SI}}= & \frac{\mu_{N}^{2}}{2916 \pi v_{D}^{4}}\left(8 f_{\mathrm{TG}}^{(N)} \frac{m_{\chi_{2}} m_{N}}{m_{H_{3}^{0}}^{2}}-27 g_{D} \sin \alpha \frac{v_{D}^{2}\left(m_{\tilde{K}}^{2}-m_{\tilde{Z}}^{2}\right)}{m_{\tilde{K}}^{2} m_{\tilde{Z}}^{2}}\right. \\
& \left.\times\left(f_{n}\left(1-\frac{Z}{A}\right)+f_{p} \frac{Z}{A}\right)\right)^{2},
\end{aligned}
$$

where $\mu_{N}^{2}=m_{\chi_{2}} m_{N} /\left(m_{\chi_{2}}+m_{N}\right)$ is the DM-nucleon reduced mass. From Eq. (80), we see that $\tilde{K}$ and $\tilde{Z}$ contribution cancels each other due to mass mixing effect. In our benchmark point $g_{D}=0.36, \sin \alpha=-0.03$, and $m_{\tilde{K}}=115 \mathrm{GeV}$, with $\left|f_{p}\right| \ll\left|f_{n}\right|$ and $f_{n}<0$, we found that the scalar mediated amplitude and vector mediated amplitude interfere destructively. If $m_{H_{3}^{0}} \sim v_{D}$, then the vector contribution dominates, and $\sigma_{N}^{\mathrm{SI}}$ does not depend on $m_{H_{3}^{0}}$ and $m_{\chi_{2}}$, and is around $5 \times 10^{-44} \mathrm{~cm}^{2}$ for our benchmark scattering with Xenon. Note that this contribution would be even larger, but thanks to the cancellation between $\tilde{K}$ and $\tilde{Z}$, the vector contribution gets a reduction of about $1 / 25$ already. The current limits on $\sigma_{N}^{\mathrm{SI}}$ are from PANDAX-II, LUX, and Xenon1T [57,74,75], and for DM mass around $10-100 \mathrm{GeV}$ is of the order of a few $10^{-46} \mathrm{~cm}^{2}$. Therefore, in order to satisfy the DD bounds, a cancellation between the vector and scalar contributions is required. We show the constraint from Xenon1T in the right panel of Fig. 4. The green area is excluded by Xenon1T with our benchmark point. For the values allowed by indirect detection, $m_{\chi_{2}}=236 \mathrm{GeV}$, the allowed region for $m_{H_{3}^{0}}$ is $103-116 \mathrm{GeV}$. Therefore, if $\chi_{2}$ is a Dirac fermion, we need to tune the mass of $H_{3}^{0}$ to avoid direct detection limit with a level of $\sim 10 \%$ tuning in mass.

\section{DM searches at the $\mathrm{LHC}$}

We start by analyzing DM searches at the LHC with mono-jet process $p p \rightarrow j \tilde{K} \rightarrow j\left(\chi_{2} \bar{\chi}_{2}\right)$. The cross section is $\sigma(j \tilde{K}) \times \operatorname{BR}\left(\tilde{K} \rightarrow \chi_{2} \bar{\chi}_{2}\right)$ for on-shell $\tilde{K}$ production if $m_{\chi_{2}}<m_{\tilde{K}} / 2$, or $\sigma\left(p p \rightarrow j \chi_{2} \bar{\chi}_{2}\right)$ which is suppressed by 3 body phase space. We first consider the constraint when $m_{\chi_{2}}<m_{\tilde{K}} / 2$, which is, however, in tension with indirect detection limits. Then the branching ratio of $\tilde{K} \rightarrow \chi_{2} \bar{\chi}_{2}$ varies from 0.14 to 0 when the mass $m_{\chi_{2}}$ is varied from $m_{\chi_{2}}=0$ to $m_{\tilde{K}} / 2$. Taking a benchmark point $m_{\tilde{K}}=$ $115 \mathrm{GeV}, g_{D}=0.36$, then the cross section of $p p \rightarrow j \tilde{K}$ at $13 \mathrm{TeV}$ LHC is $1.3 \times 10^{3} \mathrm{pb}$, and at $8 \mathrm{TeV}$ LHC is $960 \mathrm{pb}$. Then we consider the jet plus MET constraints from LHC $13 \mathrm{TeV}$ data with integrated luminosity $36 \mathrm{fb}^{-1}$ $[58,76,77]$. The ATLAS collaboration [76] studied the mono-jet limits for vector and axial vector mediator between SM quarks and DM. Their inclusive region (IM1) requires $\mathbb{E}_{T}>250 \mathrm{GeV}$ which gives 95\% C.L. constraints on cross section smaller than $0.53 \mathrm{pb}$. We calculated parton level process $j+\tilde{K}$ with a requirement $P_{t}^{j}>250 \mathrm{GeV}$, leading to cross section of about $2.4 \mathrm{pb}$ at
$13 \mathrm{TeV}$. Then we obtain a constraint on the branching ratio $\operatorname{BR}\left(\tilde{K} \rightarrow \chi_{2} \bar{\chi}_{2}\right)<0.22$, which is always satisfied in the low mass region of $m_{\chi_{2}}$. The limit is given in Fig. 4 as gray dashed line, showing that $m_{\chi_{2}} \lesssim 10 \mathrm{GeV}$ is excluded.

Aside from monojet process, the mono-X $(X=A / W / Z)$ processes are also interesting to look for. However, in s-channel vector mediator type models, usually the monojet channel provides the strongest limits [78]. Multijets plus missing energy processes have been considered in addition to monojet channel to constrain DM simplified models. The usual expectation is that the two type of constraints have comparable limits, which is the case for s-channel vector mediator type models, [79]. This is different from scalar and pseudo-scalar mediators with couplings to quarks which are proportional to quark masses, for which multi-jets process provides stronger limits [80]. The reason is that the production of scalar mediators is typically dominated by gluon fusion, which leads to more events with higher jet multiplicity [81-83].

For the other two CMS multijet plus MET searches $[58,77]$, the constraints should provide similar limits as mono-jet searches [79]. For the case with no b-tagging, we have checked the signal bin 1 and 2 in Table B.1 of Ref. [77]. However, we found the constraint is weaker than mono-jet search [76], probably because this is a parton level estimation. Adding parton shower and detector simulation should bring a conclusion close to Ref. [79]. Given the $K_{\mu}$ are not universally coupled to all quarks but couple specifically with b quark and c quark, it is natural to pay special attention to signal regions with $b$-jet tagging. The CMS sbottom search [58] looks for dijet plus MET with b-tagging. The most prominent production mode in our model is a single bottom quark in association with $\tilde{K}$ that correspond to what is called the "compressed" search region. We checked the two Bins with $\not{ }_{T}$ within $[250,300]$ and $[300,500]$ with $1 \mathrm{~b}$-jet and $H_{T}^{b}<100 \mathrm{GeV}$ requirements. The $95 \%$ C.L. limits on the cross section are about 14 and $18 \mathrm{fb}$. We calculated the cross section from parton level analysis respectively, and the corresponding cross sections after cut are 7 and $8 \mathrm{fb}$ for our benchmark $m_{\tilde{K}}=115 \mathrm{GeV}$, respectively. Therefore it does not provide an efficient constraint on $\operatorname{BR}\left(\tilde{K} \rightarrow \chi_{2} \bar{\chi}_{2}\right)$. For the CMS multi-jet plus MET search [77], we have checked the btagging signal bins $11,12,21$, and 22 which has $N_{\text {b-jet }}=1$, 2 , and found the sbottom search induced constraints [58] improve but are still weaker than the ones coming from mono-jet searches [76].

Then we consider the case with $m_{\chi_{2}}>m_{\tilde{K}} / 2$. The largest cross section that may be obtained when $m_{\tilde{K}}=115 \mathrm{GeV}$ is for $m_{\chi_{2}} \simeq 58 \mathrm{GeV}$. We get off-shell $\tilde{K}$ produced $j \chi_{2} \bar{\chi}_{2}$ cross section to be $\sim 0.01 \mathrm{pb}$ for $g_{D}=0.36$ and $\sin \alpha=-0.03$, where we only cut on $p_{T}^{j}>250 \mathrm{GeV}$. This is safe from the constraints at $13 \mathrm{TeV}$ LHC [76] that cross section after all cuts should be smaller than $0.57 \mathrm{pb}$. When $m_{\chi_{2}}$ is larger, the 
limits are even weaker due to smaller cross section. We also check the process $j j \chi_{2}^{\prime} \bar{\chi}_{2}^{\prime}{ }_{2}$ with our benchmark setup and found it is even safer from Ref. [58]. For $m_{\chi_{2}}>m_{\tilde{K}} / 2$, it is in general safe from the limits, due to small heavy quark PDF, 3-body phase space and off-shell suppression.

\section{B. Majorana dark matter}

If the mass of dark matter has contribution from a Majorana mass, the interactions between $\chi_{2}^{\prime}$ and other particles are listed in Eq. (75). In this section, we will discuss the phenomenology of such Majorana DM, including constraints from dark matter relic abundance, direct detection, indirect detection, and collider searches.

\section{DM annihilation}

The dominant annihilation process of $\chi_{2}^{\prime} \bar{\chi}^{\prime}{ }_{2} \rightarrow f \bar{f}$ is also mediated by $\tilde{K}$, after considering the mixing angle and Yukawa coupling suppression. The annihilation cross section is

$$
\begin{aligned}
(\sigma v)_{\substack{\chi_{2}^{\prime} \chi^{\prime} \rightarrow \bar{q} q \\
q=b, c}} & \frac{g_{D}^{4} c_{\chi_{2}}^{2} \sqrt{1-\frac{4 m_{q}^{2}}{s}}}{4 \pi\left[\left(s-m_{\tilde{K}}^{2}\right)^{2}+m_{\tilde{K}}^{2} \Gamma_{\tilde{K}}^{2}\right]}\left\{\left(s-4 m_{\tilde{\chi}_{2}}^{2}\right)\right. \\
& \left.+m_{q}^{2}\left[2 m_{\chi_{2}^{\prime}}^{2}\left(\frac{5 m_{\tilde{K}}^{4}-6 m_{\tilde{K}}^{2} s+3 s^{2}}{s m_{\tilde{K}}^{4}}\right)-1\right]\right\}
\end{aligned}
$$

From the annihilation formula in Eq. (81), we can find out there are two contributions, one is p-wave suppressed which is proportional to $\left(s-4 m_{\tilde{\chi}_{2}}^{2}\right) \propto m_{\chi_{2}^{\prime}}^{2} \vec{v}^{2}$, the other is helicity suppressed proportional to the quark mass. If we want to consider the annihilation cross section at freeze out, when the temperature is around $T_{f} \sim m_{\chi_{2}^{\prime}} / 20$, the dominant contribution comes from $m_{\chi_{2}^{\prime}}^{2} \vec{v}^{2}$ term. After thermal averaging, we find out the necessary dark matter mass to obtain the observed relic abundance is about 22 or $142 \mathrm{GeV}$, as shown in orange lines in Fig. 6. Note this result is for large mass splitting $M_{m} \gg m_{\chi_{2}}$. If $\Delta m \equiv m_{\eta_{1}}-m_{\eta_{2}}$ is within $\Delta m / m_{\eta_{2}} \lesssim T_{f} / m_{\eta_{2}} \sim 0.05$, the co-annihilation with $\eta_{1}$ will give a result close to Dirac DM result.

\section{DM indirect detection}

From the annihilation cross section listed in Eq. (81), both contribution from p-wave and quark mass terms are very tiny for annihilation today. Therefore, there is no constraint from indirect detection.

\section{DM direct detection}

Comparing the Lagrangian between Dirac case, Eq. (65), and Majorana case, Eq. (75), we find out the coupling of dark matter to gauge bosons $\tilde{K}$ and $\tilde{Z}$ are different. In the Majorana case, the vector coupling becomes $\gamma_{\mu} \gamma_{5}$

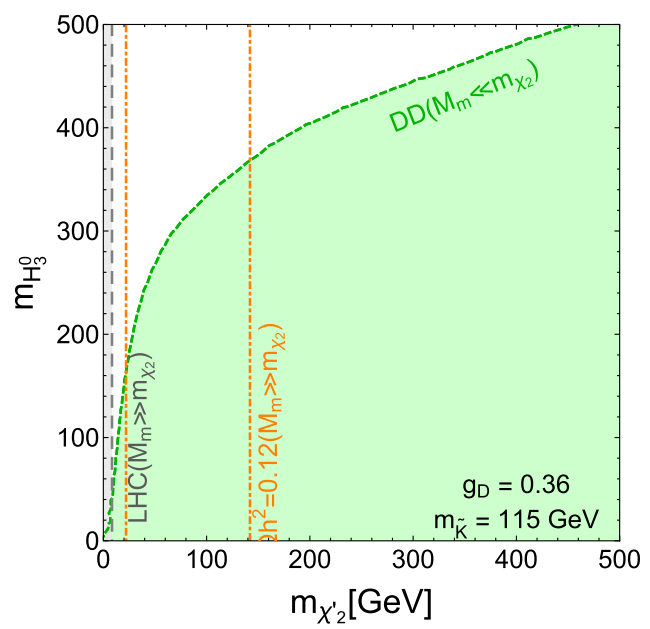

FIG. 6. The constraints for Majorana dark matter from LHC searches, direct detection and relic abundance. The labels are similar as in Fig. 4.

which induces spin dependent (SD) interaction, or velocity (momentum transfer) suppressed SI interaction. Therefore, the cross section of vector mediated processes is very small and can be ignored. There are vector coupling between DM $\eta_{2}$ and its excited state $\eta_{1}$, but it will be irrelevant if mass splitting is larger than $\mathcal{O}(100) \mathrm{keV}$. For the scalar part, if $M_{m} \gg m_{\chi_{2}}, s_{\chi_{2}}=-m_{\chi_{2}} / M_{m}$ is very tiny so we can ignore the cross section. Therefore, there are no constraints from direct detection. In the small splitting case $M_{m} \ll m_{\chi_{2}}$, $s_{\chi_{2}} \sim c_{\chi_{2}} \sim 1 / \sqrt{2}$, then the coupling between $\chi_{2}^{\prime}$ and Higgs are similar as in the Dirac case, and hence the scattering cross section between $\chi_{2}^{\prime}$ and nucleon is

$$
\sigma_{N}^{\mathrm{SI}}=\frac{16 \mu_{N}^{2}}{729 \pi v_{D}^{4}}\left(f_{\mathrm{TG}}^{(N)} \frac{m_{\chi_{2}^{\prime}} m_{N}}{m_{H_{3}^{0}}^{2}}\right)^{2} .
$$

With the scattering cross section, we give the constraints on $m_{H_{3}^{0}-} m_{\chi_{2}^{\prime}}$ plane, which are shown as the green area in Fig. 6. If $m_{H_{3}^{0}}$ is large enough, the cross-section will be very tiny.

\section{DM search at $\mathrm{LHC}$}

As previously discussed, the search for dark matter at LHC is dominantly via the interaction between dark matter and $\tilde{K}$. The branching ratio of $\operatorname{BR}(\tilde{K}) \rightarrow \chi_{2}^{\prime} \bar{\chi}^{\prime}{ }_{2}$ goes from $1 / 7$ to 0 when $m_{\chi_{2}^{\prime}}<m_{\tilde{K}} / 2$ for $M_{m} \gg m_{\chi_{2}}$. Since the cross section of $j \tilde{K}$ and $j j \tilde{K}$ at $13 \mathrm{TeV}$ LHC does not change with respect to the Dirac case, if we consider the case $m_{\chi_{2}^{\prime}}<m_{\tilde{K}} / 2$, then the constraints on invisible decay branching ratio is the same as the Dirac case. After combining the constraints from ATLAS and CMS, we can still make use of the limit $\operatorname{BR}(\tilde{K}) \rightarrow \chi_{2}^{\prime} \bar{\chi}^{\prime}{ }_{2}<0.14$ leading to a constraint on $m_{\chi_{2}^{\prime}} \lesssim 8.5 \mathrm{GeV}$ for $M_{m} \gg m_{\chi_{2}}$. 
We show the LHC constraints as a gray area in Fig. 6, which does exclude low mass DM benchmark. For $M_{m} \ll m_{\chi_{2}}$, the branching ratio $\operatorname{BR}(\tilde{K}) \rightarrow \eta_{1,2} \eta_{1,2}$ are similar as $\operatorname{BR}(\tilde{K}) \rightarrow \chi_{2}^{\prime} \bar{\chi}_{2}^{\prime}$ for $M_{m} \gg m_{\chi_{2}}$, where each channel contributes approximately $1 / 4$. But for sizable mass splitting between $\eta_{1}$ and $\eta_{2}$, the limits will be weaker because some channels $\eta_{1} \eta_{2}$ or $\eta_{1} \eta_{1}$ may not be kinematically accessible.

If $m_{\chi_{2}^{\prime}}>m_{\tilde{K}} / 2$, for the search of jet + MET at ATLAS, we compare our cross-section $\sim 0.01 \mathrm{pb}$ after cut $p_{T}^{j}>250 \mathrm{GeV}$ to the constraint at $13 \mathrm{TeV}$ LHC which is $0.57 \mathrm{pb}$ [76]. It shows that off-shell $\tilde{K}$ is very safe from limits from mono-jet searches. We also check the process $j j \chi_{2}^{\prime} \bar{\chi}_{2}{ }_{2}$ with our benchmark setup and found it is even safer from the constraints obtained in Ref. [58].

\section{CONCLUSIONS}

In this article we have studied a gauge extension of the SM that allows to explained the observed deviation of the forward backward asymmetry of the bottom-quark with respect to the expected value in SM. The new gauge boson should be neutral and should couple to only right-handed bottom and charm quarks at tree-level. Coupling to the other fermions are only induced via mixing, which should be small and fixed by the relation $g_{D} \sin \alpha \simeq-0.011$ in order to obtain the right modification of the right-handed bottom quark $Z$ coupling. Additional Higgs states are necessary for the realization of this scenario, but their signatures are too weak to ensure detection at the LHC. Moreover, we showed that, provided the new gauge boson mass is about $\sim 115 \mathrm{GeV}$ this model is consistent with all experimental constraints. The new gauge boson decays mostly into bottom and charm jets and could provide an explanation of an observed dijet excess in boosted topologies at the CMS experiment.

Cancellation of anomalies in this model leads to the presence of a charged, vectorlike lepton singlet state, as well as a vectorlike neutral state that serves as a good DM candidate. If it is a pure Dirac fermion, we can obtain the right relic abundance when its mass is around 14 or $236 \mathrm{GeV}$. The indirect detection for DM annihilation induced gamma-rays rules out the low mass DM benchmark, but keeps the high mass benchmark intact. The direct detection excludes the heavy Dirac $\chi_{2}$ benchmarks, unless a $10 \%$ fine tuning in $H_{3}^{0}$ mass is applied. If $\chi_{2}$ is split into two Majorana fermions, as is naturally the case, the direct detection constraint is easily evaded for large enough $m_{H_{3}^{0}}>400 \mathrm{GeV}$. We also can get the right relic abundance for a mass $m_{\chi_{2}^{\prime}} \sim 22$ or $142 \mathrm{GeV}$. There are no indirect detection limits because the annihilation cross section at low temperatures is highly suppressed. The LHC searches does not rule out the both DM benchmark points, but is marginal for low mass DM benchmark point.

\section{ACKNOWLEDGMENTS}

Work at University of Chicago is supported in part by U.S. Department of Energy Grant No. DE-FG0213ER41958. Work at ANL is supported in part by the U.S. Department of Energy under Contract No. DE-AC02$06 \mathrm{CH} 11357$. The work of $\mathrm{C}$. W. was partially performed at the Aspen Center for Physics, which is supported by National Science Foundation Grant No. PHY-1607611. We would like to thank Zhen Liu, LianTao Wang for useful discussions and comments. J. L. acknowledges support by an Oehme Fellowship.

\section{APPENDIX A: $C P$-EVEN HIGGS MASSES AND MIXING MATRIX}

In this section, we list a more detailed expression for $C P$-even Higgs mass and mixing matrix. The mass eigenvalues for $C P$-even Higgs in small $\cot \beta \equiv v_{1} / v_{2}$ and $\lambda_{6}$ expansion are given below,

$$
\begin{aligned}
& m_{H_{1}^{0}}^{2}=-\frac{\mu_{8} v_{D} \tan \beta}{\sqrt{2}}-\cot \beta \frac{\mu_{8}\left(v_{2}^{2}+v_{D}^{2}\right)}{\sqrt{2} v_{D}}+2\left(\lambda_{2}+\lambda_{3}\right) \cot ^{2} \beta v_{2}^{2}, \\
& m_{H_{2}^{0}}^{2}=2 \lambda_{2} v_{2}^{2}+\cot ^{2} \beta v_{2}^{2}\left(\frac{\mu_{8}^{2}}{\lambda_{2} v_{2}^{2}-\lambda_{3} v_{D}^{2}}-2 \lambda_{2}\right)+\lambda_{6}^{2} \frac{v_{2}^{2} v_{D}^{2}}{2 \lambda_{2} v_{2}^{2}-2 \lambda_{3} v_{D}^{2}}+\lambda_{6} \cot \beta \frac{\sqrt{2} \mu_{8} v_{D} v_{2}^{2}}{\lambda_{2} v_{2}^{2}-\lambda_{3} v_{D}^{2}} \\
& m_{H_{3}^{0}}^{2}=2 \lambda_{3} v_{D}^{2}+\cot ^{2} \beta v_{2}^{2}\left(\frac{-\mu_{8}^{2}}{\lambda_{2} v_{2}^{2}-\lambda_{3} v_{D}^{2}}-2 \lambda_{3}\right)-\lambda_{6}^{2} \frac{v_{2}^{2} v_{D}^{2}}{2 \lambda_{2} v_{2}^{2}-2 \lambda_{3} v_{D}^{2}}-\lambda_{6} \cot \beta \frac{\sqrt{2} \mu_{8} v_{D} v_{2}^{2}}{\lambda_{2} v_{2}^{2}-\lambda_{3} v_{D}^{2}}
\end{aligned}
$$

The mixing matrix in Eq. (29) are given in the more detailed expressions below, 


$$
\begin{aligned}
& U_{11}=1-\cot ^{2} \beta \frac{v_{2}^{2}+v_{D}^{2}}{2 v_{D}^{2}} \\
& U_{12}=\cot \beta+\mathcal{O}\left(\cot ^{2} \beta\right)+\mathcal{O}\left(\cot \beta \lambda_{6}\right) \\
& U_{13}=\cot \beta \frac{v_{2}}{v_{D}}+\mathcal{O}\left(\cot ^{2} \beta\right)+\mathcal{O}\left(\cot \beta \lambda_{6}\right) \\
& U_{22}=1+\cot ^{2} \beta\left(-\frac{1}{2}-\frac{\mu_{8}^{2} v_{2}^{2}}{4\left(\lambda_{2} v_{2}^{2}-\lambda_{3} v_{D}^{2}\right)^{2}}\right)-\lambda_{6} \cot \beta \frac{\mu_{8} v_{D} v_{2}^{2}}{2 \sqrt{2}\left(\lambda_{2} v_{2}^{2}-\lambda_{3} v_{D}^{2}\right)^{2}}-\lambda_{6}^{2} \frac{v_{2}^{2} v_{D}^{2}}{8\left(\lambda_{2} v_{2}^{2}-\lambda_{3} v_{D}^{2}\right)^{2}} \\
& U_{23}=-\cot \beta \frac{\mu_{8} v_{2}}{\sqrt{2}\left(\lambda_{2} v_{2}^{2}-\lambda_{3} v_{D}^{2}\right)}-\lambda_{6} \frac{v_{2} v_{D}}{2\left(\lambda_{2} v_{2}^{2}-\lambda_{3} v_{D}^{2}\right)}+\mathcal{O}\left(\cot ^{2} \beta\right) \\
& U_{33}=1-\cot ^{2} \beta \frac{v_{2}^{2}}{4}\left(\frac{\mu_{8}^{2}}{\left(\lambda_{2} v_{2}^{2}-\lambda_{3} v_{D}^{2}\right)^{2}}+\frac{2}{v_{D}^{2}}\right)-\lambda_{6}^{2} \frac{v_{2}^{2} v_{D}^{2}}{8\left(\lambda_{2} v_{2}^{2}-\lambda_{3} v_{D}^{2}\right)^{2}}-\lambda_{6} \tan \beta \frac{\mu_{8} v_{D} v_{2}^{2}}{2 \sqrt{2}\left(\lambda_{2} v_{2}^{2}-\lambda_{3} v_{D}^{2}\right)^{2}},
\end{aligned}
$$

where the $U$ matrix is approximate antisymmetric that $U_{21} \sim-U_{12}, U_{31} \sim-U_{13}$, and $U_{23} \sim-U_{32}$.

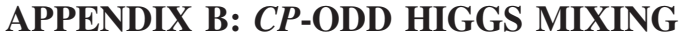 MATRIX AND INTERACTIONS}

The mass matrix of $C P$-odd Higgs are given in Eq. (23) and the mass of $A^{0}$ is given in Eq. (24). It is straightforward to calculate the mixing matrix $U_{\text {odd }}$,

$$
\left(\begin{array}{l}
a_{1}^{0} \\
a_{2}^{0} \\
a_{3}^{0}
\end{array}\right)=U_{\text {odd }}\left(\begin{array}{l}
H_{1}^{0} \\
H_{2}^{0} \\
H_{3}^{0}
\end{array}\right)=\left(\begin{array}{ccc}
\frac{v_{D} v_{2}}{\bar{v}^{2}} & \frac{v_{1}}{v} & -\frac{v_{2}}{v} \frac{v_{1} v_{2}}{\bar{v}^{2}} \\
-\frac{v_{D} v_{1}}{\bar{v}^{2}} & \frac{v_{2}}{v} & \frac{v_{1}}{v} \frac{v_{1} v_{2}}{\bar{v}^{2}} \\
\frac{v_{1} v_{2}}{\bar{v}^{2}} & 0 & \frac{v_{D} v}{\bar{v}^{2}}
\end{array}\right)\left(\begin{array}{c}
A^{0} \\
G_{2}^{0} \\
G_{3}^{0}
\end{array}\right),
$$

where $\bar{v}^{2} \equiv\left(v_{1}^{2} v_{2}^{2}+v_{1}^{2} v_{D}^{2}+v_{2}^{2} v_{D}^{2}\right)^{1 / 2}$. At leading order $c_{\beta}$ approximation, $U_{\text {odd }}$ becomes

$$
U_{\text {odd }} \approx\left(\begin{array}{ccc}
1-\frac{c_{\beta}^{2}\left(v^{2}+v_{D}^{2}\right)}{2 v_{D}^{2}} & c_{\beta} & -\frac{c_{\beta} v}{v_{D}} \\
-c_{\beta} & 1-c_{\beta}^{2} & \frac{c_{\beta}^{2} v}{v_{D}} \\
\frac{c_{\beta} v}{v_{D}} & 0 & 1-\frac{c_{\beta}^{2} v^{2}}{2 v_{D}^{2}}
\end{array}\right),
$$

and in this limit $G_{2}^{0}$ and $G_{3}^{0}$ are eaten by $Z$ and $K$ respectively. The interactions between $A^{0}$ and bottom and top quarks are

$$
\begin{aligned}
\mathcal{L}_{\mathrm{tb}}^{A^{0}}= & -i \frac{m_{t}}{t_{\beta} v} \bar{t}_{L} t_{R} A^{0}+i \frac{m_{\tilde{b}}}{t_{\beta} v}\left(c_{b, L}-c_{b, R} \frac{s_{\beta} v^{2}}{v_{D}^{2}}\right) \overline{\tilde{b}}_{L} \tilde{b}_{R} A^{0} \\
& \times i \frac{m_{\tilde{b}}}{t_{\beta} v}\left(c_{b, L} \frac{c_{b, R}}{s_{b, R}}+\frac{v^{2}}{v_{D}^{2}} s_{\beta} s_{b, R}\right) \overline{\tilde{b}}_{L} \tilde{\psi}_{b, R} A^{0} \\
& -i \frac{m_{\tilde{b}}}{t_{\beta} v}\left(\frac{c_{b, L}}{s_{b, L}} c_{b, R} \frac{v^{2}}{v_{D}^{2}} s_{\beta}+s_{b, L}\right) \overline{\tilde{\psi}}_{b, L} \tilde{b}_{R} A^{0} \\
& \times i \frac{m_{\tilde{b}}}{t_{\beta} v}\left(c_{b, L} \frac{s_{b, R}}{s_{b, L}} \frac{v^{2}}{v_{D}^{2}}-c_{b, R} \frac{s_{b, L}}{s_{b, R}}\right) \tilde{\tilde{\psi}}_{b, L} \tilde{\psi}_{b, R} A^{0}+\text { H.c. },
\end{aligned}
$$

where we see the dominant interaction is with $\bar{t} t$ only suppressed by $c_{\beta}$. The interactions between $A^{0}$ and charm and strange quarks are similar,

$$
\begin{aligned}
\mathcal{L}_{\mathrm{cs}}^{A^{0}}= & i \frac{m_{s}}{t_{\beta} v} \bar{s}_{L} s_{R} A^{0}-i \frac{m_{\tilde{c}}}{t_{\beta} v}\left(c_{c, L}-c_{c, R} \frac{s_{\beta} v^{2}}{v_{D}^{2}}\right) \overline{\tilde{c}}_{L} \tilde{c}_{R} A^{0} \\
& -i \frac{m_{\tilde{c}}}{t_{\beta} v}\left(c_{c, L} \frac{c_{c, R}}{s_{c, R}}+\frac{v^{2}}{v_{D}^{2}} s_{\beta} s_{c, R}\right) \overline{\tilde{c}}_{L} \tilde{\psi}_{c, R} A^{0} \\
& \times i \frac{m_{\tilde{c}}}{t_{\beta} v}\left(\frac{c_{c, L}}{s_{c, L}} c_{c, R} \frac{v^{2}}{v_{D}^{2}} s_{\beta}+s_{c, L}\right) \overline{\tilde{\psi}}_{c, L} \tilde{c}_{R} A^{0} \\
& -i \frac{m_{\tilde{c}}}{t_{\beta} v}\left(c_{c, L} \frac{s_{c, R}}{s_{c, L}} \frac{v^{2}}{v_{D}^{2}}-c_{c, R} \frac{s_{c, L}}{s_{c, R}}\right) \overline{\tilde{\psi}}_{c, L} \tilde{\psi}_{c, R} A^{0}+\text { H.c. },
\end{aligned}
$$

where there is a minus difference between $t b$ and $c s$ quarks from $\Phi_{2}$ and $\tilde{\Phi}_{2}$. We see $A^{0}$ can decay to SM top, bottom, charm and strange quark pair with width proportional to $m_{q}^{2} c_{\beta}^{2}$ in the leading terms. 
[1] G. Aad et al. (ATLAS Collaboration), Observation of a new particle in the search for the Standard Model Higgs boson with the ATLAS detector at the LHC, Phys. Lett. B 716, 1 (2012).

[2] S. Chatrchyan et al. (CMS Collaboration), Observation of a new boson at a mass of $125 \mathrm{GeV}$ with the CMS experiment at the LHC, Phys. Lett. B 716, 30 (2012).

[3] S. Schael et al. , Precision electroweak measurements on the $Z$ resonance, Phys. Rep. 427, 257 (2006).

[4] G. Bertone, D. Hooper, and J. Silk, Particle dark matter: Evidence, candidates and constraints, Phys. Rep. 405, 279 (2005).

[5] D. Choudhury, T. M. P. Tait, and C. E. M. Wagner, Beautiful mirrors and precision electroweak data, Phys. Rev. D 65, 053002 (2002).

[6] D. E. Morrissey and C.E. M. Wagner, Beautiful mirrors, unification of couplings and collider phenomenology, Phys. Rev. D 69, 053001 (2004).

[7] K. Kumar, W. Shepherd, T. M. P. Tait, and R. Vega-Morales, Beautiful mirrors at the LHC, J. High Energy Phys. 08 (2010) 052.

[8] B. Batell, S. Gori, and L.-T. Wang, Higgs couplings and precision electroweak data, J. High Energy Phys. 01 (2013) 139.

[9] X.-G. He and G. Valencia, The $Z \rightarrow b \bar{b}$ decay asymmetry and left-right models, Phys. Rev. D 66, 013004 (2002); Erratum, Phys. Rev. D66, 079901(E) (2002).

[10] A. Djouadi, G. Moreau, and F. Richard, Resolving the $A_{\mathrm{FB}}^{b}$ puzzle in an extra dimensional model with an extended gauge structure, Nucl. Phys. B773, 43 (2007).

[11] J. Liu, X.-P. Wang, and F. Yu, A tale of two portals: Testing light, hidden new physics at future $e^{+} e^{-}$colliders, J. High Energy Phys. 06 (2017) 077.

[12] D. B. Kaplan, Flavor at SSC energies: A New mechanism for dynamically generated fermion masses, Nucl. Phys. B365, 259 (1991).

[13] G. Aad et al. (ATLAS, CMS Collaboration), Measurements of the Higgs boson production and decay rates and constraints on its couplings from a combined ATLAS and CMS analysis of the LHC pp collision data at $\sqrt{s}=7$ and $8 \mathrm{TeV}$, J. High Energy Phys. 08 (2016) 045.

[14] K. Agashe, R. Contino, L. Da Rold, and A. Pomarol, A custodial symmetry for $Z b \bar{b}$, Phys. Lett. B 641, 62 (2006).

[15] M.-x. Luo, W. Wu, and G.-h. Zhu, Unparticle physics and $A_{\mathrm{FB}}^{b}$ on the $\mathrm{Z}$ pole, Phys. Lett. B 659, 349 (2008).

[16] E. L. Berger, Q.-H. Cao, and I. Low, Model independent constraints among the Wtb, Zb anti-b, and $\mathrm{Zt}$ anti-t couplings, Phys. Rev. D 80, 074020 (2009).

[17] L. Da Rold, Solving the $A_{F B}^{b}$ anomaly in natural composite models, J. High Energy Phys. 02 (2011) 034.

[18] S. Gori, J. Gu, and L.-T. Wang, The $Z b \bar{b}$ couplings at future $e^{+} e^{-}$colliders, J. High Energy Phys. 04 (2016) 062.

[19] Tevatron Electroweak Working Group, CDF, DELPHI, SLD Electroweak and Heavy Flavour Groups, ALEPH, LEP Electroweak Working Group, SLD, OPAL, D0, L3 Collaborations, L. E. W. Group, Precision electroweak measurements and constraints on the standard model, arXiv: 1012.2367.

[20] J. Alcaraz, Precision electroweak measurements and constraints on the standard model, arXiv:0911.2604.
[21] J. Alcaraz et al., A combination of preliminary electroweak measurements and constraints on the standard model, arXiv: hep-ex/0612034.

[22] SLD Electroweak Group, SLD Heavy Flavor Group, DELPHI, LEP, ALEPH, OPAL, LEP Electroweak Working Group, L3 Collaboration, t. S. Electroweak, A combination of preliminary electroweak measurements and constraints on the standard model, arXiv:hep-ex/0312023.

[23] F. Jegerlehner and A. Nyffeler, The Muon g-2, Phys. Rep. 477, 1 (2009).

[24] D. Hanneke, S. Fogwell, and G. Gabrielse, New Measurement of the Electron Magnetic Moment and the Fine Structure Constant, Phys. Rev. Lett. 100, 120801 (2008).

[25] K. A. Olive et al. (Particle Data Group Collaboration), Review of particle physics, Chin. Phys. C 38, 090001 (2014).

[26] CMS Collaboration, Technical Report No. CMS-PAS-EXO17-001, 2017.

[27] ATLAS Collaboration, Technical Report No. ATLASCONF-2016-031, 2016.

[28] CMS Collaboration, Technical Report No. CMS-PAS-HIG16-025, 2016.

[29] G. Aad et al. (ATLAS Collaboration), Search for the neutral Higgs bosons of the minimal supersymmetric standard model in $p p$ collisions at $\sqrt{s}=7 \mathrm{TeV}$ with the ATLAS detector, J. High Energy Phys. 02 (2013) 095.

[30] M. Aaboud et al. (ATLAS Collaboration), Search for new high-mass phenomena in the dilepton final state using $36.1 \mathrm{fb}^{-1}$ of proton-proton collision data at $\sqrt{s}=13 \mathrm{TeV}$ with the ATLAS detector, J. High Energy Phys. 10 (2017) 182.

[31] G. Aad et al. (ATLAS Collaboration), Search for high mass dilepton resonances in $p p$ collisions at $\sqrt{s}=7 \mathrm{TeV}$ with the ATLAS experiment, Phys. Lett. B 700, 163 (2011).

[32] G. Aad et al. (ATLAS Collaboration), Search for high-mass dilepton resonances in pp collisions at $\sqrt{s}=8 \mathrm{TeV}$ with the ATLAS detector, Phys. Rev. D 90, 052005 (2014).

[33] ATLAS Collaboration, Technical Report No. ATLASCONF-2016-029, 2016.

[34] R. Hooper and G. Landsberg (D0 Collaboration), Technical Report No. D0-4577, 2004.

[35] T. Aaltonen et al. (CDF Collaboration), Search for New Physics in High Mass Electron-Positron Events in $p \bar{p}$ Collisions at $\sqrt{s}=1.96-\mathrm{TeV}$, Phys. Rev. Lett. 99, 171802 (2007).

[36] R. Aaij et al. (LHCb Collaboration), Test of Lepton Universality Using $B^{+} \rightarrow K^{+} \ell^{+} \ell^{-}$Decays, Phys. Rev. Lett. 113, 151601 (2014).

[37] R. Aaij et al. (LHCb Collaboration), Test of lepton universality with $B^{0} \rightarrow K^{* 0} \ell^{+} \ell^{-}$decays, J. High Energy Phys. 08 (2017) 055.

[38] J. P. Lees et al. (BABAR Collaboration), Evidence for an Excess of $\bar{B} \rightarrow D^{(*)} \tau^{-} \bar{\nu}_{\tau}$ Decays, Phys. Rev. Lett. 109, 101802 (2012).

[39] J. P. Lees et al. (BABAR Collaboration), Measurement of an excess of $\bar{B} \rightarrow D^{(*)} \tau^{-} \bar{\nu}_{\tau}$ decays and implications for charged Higgs bosons, Phys. Rev. D 88, 072012 (2013).

[40] R. Aaij et al. (LHCb Collaboration), Measurement of the Ratio of Branching Fractions $\mathcal{B}\left(\bar{B}^{0} \rightarrow D^{*+} \tau^{-} \bar{\nu}_{\tau}\right) /$ $\mathcal{B}\left(\bar{B}^{0} \rightarrow D^{*+} \mu^{-} \bar{\nu}_{\mu}\right)$, Phys. Rev. Lett. 115, 111803 (2015); Publisher's Note, Phys. Rev. Lett. 115, 159901 (2015). 
[41] M. Huschle et al. (Belle Collaboration), Measurement of the branching ratio of $\bar{B} \rightarrow D^{(*)} \tau^{-} \bar{\nu}_{\tau}$ relative to $\bar{B} \rightarrow D^{(*)} \ell^{-} \bar{\nu}_{\ell}$ decays with hadronic tagging at Belle, Phys. Rev. D 92, 072014 (2015).

[42] Y. Sato et al. (Belle Collaboration), Measurement of the branching ratio of $\bar{B}^{0} \rightarrow D^{*+} \tau^{-} \bar{\nu}_{\tau}$ relative to $\bar{B}^{0} \rightarrow$ $D^{*+} \ell^{-} \bar{\nu}_{\ell}$ decays with a semileptonic tagging method, Phys. Rev. D 94, 072007 (2016).

[43] R. Aaij et al. (LHCb Collaboration), Measurement of the $B^{0} \rightarrow D^{*-} \tau^{+} \nu_{\tau}$ branching fraction using three-prong $\tau$ decays, arXiv:1711.02505.

[44] J. F. Kamenik, Y. Soreq, and J. Zupan, Lepton flavor universality violation without new sources of quark flavor violation, Phys. Rev. D 97, 035002 (2018).

[45] F. Sala and D. M. Straub, A new light particle in B decays?, Phys. Lett. B 774, 205 (2017).

[46] A. Alloul, N. D. Christensen, C. Degrande, C. Duhr, and B. Fuks, FeynRules 2.0-A complete toolbox for tree-level phenomenology, Comput. Phys. Commun. 185, 2250 (2014).

[47] J. Alwall, R. Frederix, S. Frixione, V. Hirschi, F. Maltoni, O. Mattelaer, H. S. Shao, T. Stelzer, P. Torrielli, and M. Zaro, The automated computation of tree-level and next-toleading order differential cross sections, and their matching to parton shower simulations, J. High Energy Phys. 07 (2014) 079.

[48] M. Aaboud et al. (ATLAS Collaboration), Search for the standard model Higgs boson produced by vector-boson fusion and decaying to bottom quarks in $\sqrt{s}=8 \mathrm{TeV}$ pp collisions with the ATLAS detector, J. High Energy Phys. 11 (2016) 112.

[49] ATLAS Collaboration, Technical Report No. ATLASCONF-2016-063, 2016.

[50] G. Rauco (CMS Collaboration), in 3rd Large Hadron Collider Physics Conference (LHCP 2015) St. Petersburg, Russia, 2015 (to be published).

[51] M. Aaboud et al. (ATLAS Collaboration), Evidence for the $H \rightarrow b \bar{b}$ decay with the ATLAS detector, J. High Energy Phys. 12 (2017) 024.

[52] CMS Collaboration, Technical Report No. CMS-PAS-HIG17-010, 2017.

[53] D. Tucker-Smith and N. Weiner, Inelastic dark matter, Phys. Rev. D 64, 043502 (2001).

[54] P. A. R. Ade et al. (Planck Collaboration), Planck 2015 results. XIII. Cosmological parameters, Astron. Astrophys. 594, A13 (2016).

[55] M. Ackermann et al. (Fermi-LAT Collaboration), Searching for Dark Matter Annihilation from Milky Way Dwarf Spheroidal Galaxies with Six Years of Fermi Large Area Telescope Data, Phys. Rev. Lett. 115, 231301 (2015).

[56] A. Albert et al. (DES, Fermi-LAT Collaboration), Searching for dark matter annihilation in recently discovered Milky Way satellites with Fermi-LAT, Astrophys. J. 834, 110 (2017).

[57] E. Aprile et al. (XENON Collaboration), First Dark Matter Search Results from the XENON1T Experiment, Phys. Rev. Lett. 119, 181301 (2017).

[58] A. M. Sirunyan et al. (CMS Collaboration), Search for the pair production of third-generation squarks with two-body decays to a bottom or charm quark and a neutralino in proton-proton collisions at sqrt(s) $=13 \mathrm{TeV}$, Phys. Lett. B 778, 263 (2018).

[59] J. A. Adams, S. Sarkar, and D. W. Sciama, CMB anisotropy in the decaying neutrino cosmology, Mon. Not. R. Astron. Soc. 301, 210 (1998).

[60] N. Padmanabhan and D. P. Finkbeiner, Detecting dark matter annihilation with $\mathrm{CMB}$ polarization: Signatures and experimental prospects, Phys. Rev. D 72, 023508 (2005).

[61] S. Galli, F. Iocco, G. Bertone, and A. Melchiorri, CMB constraints on Dark Matter models with large annihilation cross-section, Phys. Rev. D 80, 023505 (2009).

[62] T. R. Slatyer, N. Padmanabhan, and D. P. Finkbeiner, CMB constraints on WIMP annihilation: Energy absorption during the recombination epoch, Phys. Rev. D 80, 043526 (2009).

[63] T. R. Slatyer, Indirect dark matter signatures in the cosmic dark ages. I. Generalizing the bound on s-wave dark matter annihilation from Planck results, Phys. Rev. D 93, 023527 (2016).

[64] P. Ciafaloni, D. Comelli, A. Riotto, F. Sala, A. Strumia, and A. Urbano, Weak Corrections are Relevant for Dark Matter Indirect Detection, J. Cosmol. Astropart. Phys. 03 (2011) 019.

[65] M. Ackermann et al. (Fermi-LAT Collaboration), The Fermi Galactic center $\mathrm{GeV}$ excess and implications for dark matter, Astrophys. J. 840, 43 (2017).

[66] M. Ackermann et al. (Fermi-LAT Collaboration), Search for extended gamma-ray emission from the Virgo galaxy cluster with Fermi-LAT, Astrophys. J. 812, 159 (2015).

[67] P. Junnarkar and A. Walker-Loud, Scalar strange content of the nucleon from lattice QCD, Phys. Rev. D 87, 114510 (2013).

[68] R. J. Hill and M. P. Solon, Universal behavior in the scattering of heavy, weakly interacting dark matter on nuclear targets, Phys. Lett. B 707, 539 (2012).

[69] G. Belanger, F. Boudjema, A. Pukhov, and A. Semenov, MicrOMEGAs 2.0: A Program to calculate the relic density of dark matter in a generic model, Comput. Phys. Commun. 176, 367 (2007).

[70] J. M. Alarcon, J. Martin Camalich, and J. A. Oller, The chiral representation of the $\pi N$ scattering amplitude and the pion-nucleon sigma term, Phys. Rev. D 85, 051503 (2012).

[71] J. M. Alarcon, L. S. Geng, J. Martin Camalich, and J. A. Oller, The strangeness content of the nucleon from effective field theory and phenomenology, Phys. Lett. B 730, 342 (2014).

[72] F. Bishara, J. Brod, B. Grinstein, and J. Zupan, Chiral effective theory of dark matter direct detection, J. Cosmol. Astropart. Phys. 02 (2017) 009.

[73] M. Escudero, A. Berlin, D. Hooper, and M.-X. Lin, Toward (finally!) ruling out $\mathrm{Z}$ and Higgs mediated dark matter models, J. Cosmol. Astropart. Phys. 12 (2016) 029.

[74] X. Cui et al. (PandaX-II Collaboration), Dark Matter Results From 54-Ton-Day Exposure of PandaX-II Experiment, Phys. Rev. Lett. 119, 181302 (2017).

[75] D. S. Akerib et al. (LUX Collaboration), Results from a Search for Dark Matter in the Complete LUX Exposure, Phys. Rev. Lett. 118, 021303 (2017).

[76] M. Aaboud et al. (ATLAS Collaboration), Search for dark matter and other new phenomena in events with an 
energetic jet and large missing transverse momentum using the ATLAS detector, J. High Energy Phys. 01 (2018) 126.

[77] A. M. Sirunyan et al. (CMS Collaboration), Search for supersymmetry in multijet events with missing transverse momentum in proton-proton collisions at $13 \mathrm{TeV}$, Phys. Rev. D 96, 032003 (2017).

[78] A. J. Brennan, M. F. McDonald, J. Gramling, and T. D. Jacques, Collide and conquer: Constraints on simplified dark matter models using Mono-X collider searches, J. High Energy Phys. 05 (2016) 112.

[79] S. P. Liew, M. Papucci, A. Vichi, and K. M. Zurek, Mono-X versus direct searches: Simplified models for dark matter at the LHC, J. High Energy Phys. 06 (2017) 082.
[80] O. Buchmueller, S. A. Malik, C. McCabe, and B. Penning, Constraining Dark Matter Interactions with Pseudoscalar and Scalar Mediators Using Collider Searches for Multijets plus Missing Transverse Energy, Phys. Rev. Lett. 115, 181802 (2015).

[81] U. Haisch, F. Kahlhoefer, and J. Unwin, The impact of heavy-quark loops on LHC dark matter searches, J. High Energy Phys. 07 (2013) 125.

[82] P. J. Fox and C. Williams, Next-to-leading order predictions for dark matter production at hadron colliders, Phys. Rev. D 87, 054030 (2013).

[83] U. Haisch, F. Kahlhoefer, and E. Re, QCD effects in monojet searches for dark matter, J. High Energy Phys. 12 (2013) 007. 